\title{
Bubbling in a co-flow at high Reynolds numbers
}

\author{
J. M. Gordillo ${ }^{\text {a) }}$ \\ Área de Mecánica de Fluidos, Departamento de Ingeniería Aeroespacial y Mecánica de Fluidos, \\ Universidad de Sevilla, Avda. de los Descubrimientos s/n, 41092 Sevilla, Spain
}

A. Sevilla ${ }^{\text {b) }}$ and C. Martínez-Bazán ${ }^{\text {c) }}$

Área de Mecánica de Fluidos, Universidad de Jaén, Campus de las Lagunillas, 23071 Jaén, Spain

(Received 24 November 2006; accepted 2 May 2007; published online 19 July 2007)

\begin{abstract}
The physical mechanisms underlying bubble formation from a needle in a co-flowing liquid environment at high Reynolds numbers are studied in detail with the aid of experiments and boundary-integral numerical simulations. To determine the effect of gas inertia the experiments were carried out with air and helium. The influence of the injection system is elucidated by performing experiments using two different facilities, one where the constancy of the gas flow-rate entering the bubble is ensured, and another one where the gas is injected through a needle directly connected to a pressurized chamber. In the case of constant flow-rate injection conditions, the bubbling frequency has been shown to hardly depend on the gas density, with a bubble size given by $d_{b} / r_{o}$ $\simeq\left[6 U\left(k^{*} U+k_{2}\right) /(U-1)\right]^{1 / 3}$ for $U \gtrsim 2$, where $U$ is the gas-to-liquid ratio of the mean velocities, $r_{o}$ is the radius of the gas injection needle, and $k^{*}=5.84$ and $k_{2}=4.29$, with $d_{b} / r_{o} \sim 3.3 U^{1 / 3}$ for $U \gg 1$. Nevertheless, in this case the effect of gas density is relevant to describe the final instants of bubble breakup, which take place at a time scale much smaller than the bubbling time, $t_{b}$. This effect is evidenced by the liquid jets penetrating the gas bubbles upon their pinch-off. Our measurements indicate that the velocity of the penetrating jets is considerably larger in air bubbles than in helium bubbles due to the distinct gas inertia of both situations. However, in the case of constant pressure supply conditions, the bubble size strongly depends on the density of the gas through the pressure loss along the gas injection needle. Furthermore, under the operating conditions reported here, the equivalent diameters of the bubbles are between 10\% and $20 \%$ larger than their constant flow-rate counterparts. In addition, the experiments and the numerical results show that, under constant pressure supply, helium bubbles are approximately 10\% larger than air bubbles due to the gas density effect on the bubbling process. (C) 2007 American Institute of Physics.
\end{abstract}

[DOI: 10.1063/1.2747996]

\section{INTRODUCTION}

The exhaustive theoretical, numerical, and experimental effort dedicated to studying the break-up of liquid jets in air is justified not only by their importance in technological applications but also by their widespread presence in daily life. The first well known analytical study about the break-up of liquid jets was performed by Rayleigh, ${ }^{1}$ although the role of capillarity was previously described by Savart ${ }^{2}$ and Plateau. ${ }^{3}$ The stability analysis performed by Rayleigh is able to predict, with a high degree of accuracy, the wavelength or, equivalently, the volume of the main drops formed when a circular jet discharges in air. The formation of satellite drops and the description of the latest instants prior to pinch-off (see the review by Eggers ${ }^{4}$ ), or the transition from the socalled jetting regime to the dripping regime, ${ }^{5,6}$ are details extensively reported in the literature. However, although the generation of bubbles is a phenomenon as common as the drop formation, and of great relevance in a large number of industrial processes, our knowledge of the bubble formation process is still far from being fully complete despite the re-

\footnotetext{
${ }^{a}$ Electronic mail: jgordill@us.es

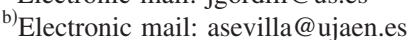

${ }^{c)}$ Electronic mail: cmbazan@ujaen.es
}

cent advances in the analytical and experimental description of the bubble pinch-off at $\operatorname{low}^{7-9}$ and high ${ }^{9-13}$ Reynolds numbers. For example, the most studied geometry, due its simplicity and importance in many chemical engineering applications, is the growth and subsequent pinch-off of a bubble from an orifice, or needle, placed at the bottom of a liquid pool. ${ }^{14-21}$ In this case, the formation of single bubbles at low and moderate gas Weber numbers is a phenomenon relatively well understood; but even such a simple situation presents difficulties not found in the drop formation problem. Indeed, the gas stream discharging from an orifice into a stagnant liquid pool always generates bubbles nearby the orifice, without forming a long ligament, because the configuration is absolutely unstable independently of the gas Weber number. ${ }^{22,23}$ In addition, if the pressure fluctuations originated by the unsteady liquid flow at the needle exit become of the order of the pressure drop along the gas injection line, there may exist a coupling effect between the feeding line and the bubble formation process ${ }^{15,19,24,25}$ and, unlike the case of liquid jets, the flow-rate exiting the needle may not be constant during the bubbling time. Therefore, the formation of bubbles is a strongly nonlinear process, coupled with the dynamics of the gas injection system.

Unfortunately the bubbles released from a needle into a 
stagnant liquid reservoir are not sufficiently small for most of the engineering applications and different configurations need to be employed to generate smaller ones, of the order of $\mathcal{O}(1-100 \mu \mathrm{m})$. Although nanobubbles can be generated through porous membranes injecting the gas at a pressure of the order of $10 \mathrm{MPa},{ }^{26}$ a common way to reduce the bubble size is to inject the gas stream within a liquid co-flow. ${ }^{27}$ This technique also avoids the coalescence of bubbles and it is nowadays extensively employed in microfluid applications. ${ }^{28}$ An example can be found in the large number of micro devices based on the so-called flow-focusing configuration, where an inner gas stream, surrounded by an outer liquid co-flow, is driven through a small orifice. ${ }^{29-34}$ In this particular configuration, dimensional analysis and physical arguments have provided with closed expressions for the scaling of the bubble size based on the different control parameters. ${ }^{29,32-36}$ However, although the proposed scaling laws are valid for practical purposes because they predict the bubble volume relatively well, a more detailed theoretical and numerical approach would be desirable to fully comprehend the bubble formation mechanisms.

Making use of numerical simulations in the zero Reynolds number limit (Stokes flow), Jensen et al. ${ }^{37}$ have recently derived scaling laws to predict the volume of bubbles formed using the flow-focusing geometry that agree with previous experimental results. ${ }^{32}$ Although a similar study in the limit of high Reynolds numbers applied to the flowfocusing geometry is still lacking, Oguz and Prosperetti ${ }^{19}$ analyzed the fundamental problem of bubble formation and detachment from a needle using a boundary-integral method. More importantly regarding the present context, they also considered the possibility of including a liquid co-flow surrounding the forming bubble. From the experimental point of view, Sevilla et al., ${ }^{23}$ and latter on Gañán et al., ${ }^{38}$ performed a series of experiments using the co-flowing geometry previously analyzed by Chuang and Goldschmidt ${ }^{27}$ and Oguz and Prosperetti ${ }^{19}$ with the aim at determining the formation mechanisms and scaling laws of air bubbles inside a laminar, high-Reynolds-number water jet discharging in a still air atmosphere. In this work, the authors identify two different regimes, namely a jetting regime and a bubbling regime, depending on the Weber number and the gas-to-liquid velocity ratio. The jetting regime, experimentally observed if the gas and liquid injection velocities are similar, is characterized by a long gas ligament that breaks far from the injection needle by the growth of external noise. On the other hand, the bubbling regime, observed when the gas stream flows sufficiently faster than the surrounding liquid, is characterized by a periodic generation of bubbles which can be naturally divided into two stages: an expansion stage and a collapse stage. ${ }^{39}$ The transition from the jetting to the bubbling regime was predicted by a change in the nature of the instability from a convective to an absolute instability. In addition, a simple description of the mechanisms governing the collapse stage is given, providing a relevant time scale that corresponds to a Kelvin-Helmholtz instability. Figure 1 shows a sketch of the periodic bubble formation process in the bubbling regime. Notice that when a bubble is released, it leaves behind an intact ligament of length $l_{i}$ attached to the (a)

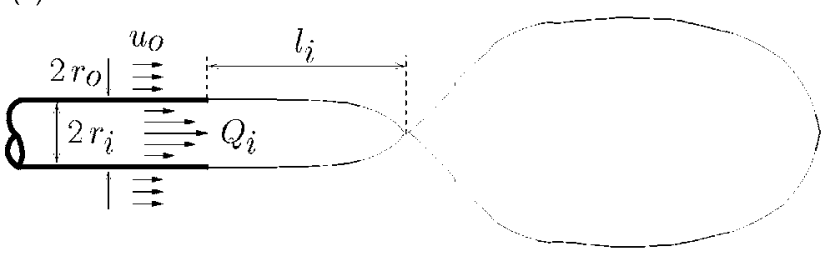

(b)
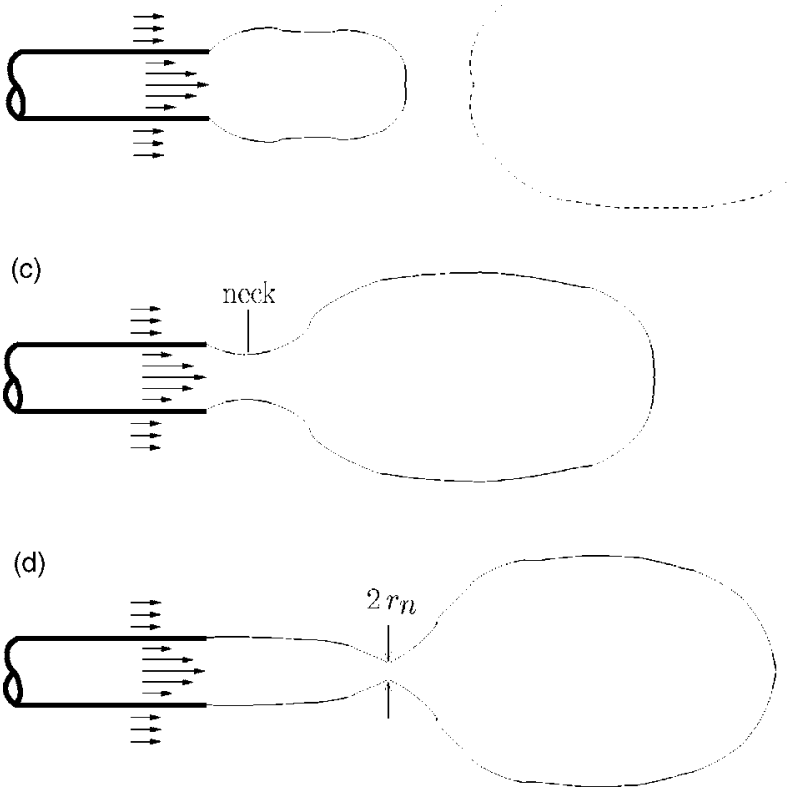

FIG. 1. Schematic representation of the bubble formation process.

injection needle [Fig. 1(a)]. The intact gas stem starts to inflate during the expansion stage, which is comprised of the detachment of a bubble [Fig. 1(a)] to the moment when a neck is formed [Fig. 1(c)]. At this point the radius of the neck, $r_{n}$, begins to decrease [Fig. 1(c)] during the collapse stage until it pinches-off forming a new bubble [Fig. 1(d)].

The main purpose of the present paper is to provide a detailed description of the entire bubble formation process using the co-flowing geometry and making use of experiments and numerical simulations. In particular, we will propose a suitable scaling for the bubbling time and bubble size as a function of the control parameters and will provide additional evidence that support the key role of the gas inertia in the collapse stage comparing the experimental results with our numerical simulations. The existing coupling between the gas injection system and the bubble growth, ${ }^{15,19,24,25}$ and subsequent collapse, will also be elucidated by performing experiments using two different facilities, with the first one designed to ensure a constant gas flow-rate feeding the bubble during the formation process.

The paper is organized as follows: The experimental facility is described in Sec. II, along with a complete discussion of the different conditions required by the injection system to ensure a constant gas flow-rate or constant pressure injection supply. The experimental results are discussed in Sec. III with a detailed comparison with the numerics. Additionally, in Sec. III we also propose a scaling law for the bubble formation frequency and bubble size as a function of 


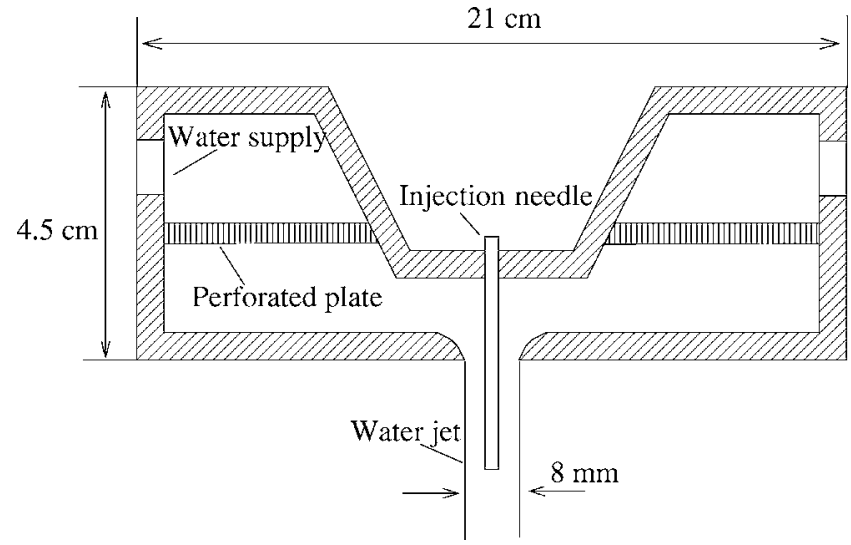

FIG. 2. Sketch of the water nozzle.

the relevant control parameters for each of the different setups used in the study. Finally, Sec. IV is devoted to conclusions and the Appendix describes the boundary integral numerical method and the numerical scheme.

\section{EXPERIMENTAL SETUP}

The experimental facility used in the present study, shown in Fig. 2, consists of a vertical water jet discharging downwards from a cylindrical reservoir of $21 \mathrm{~cm}$ in diameter and $4.5 \mathrm{~cm}$ of length through a nozzle of $8 \mathrm{~mm}$ diameter. A perforated plate and a piece of foam were placed inside the water vessel to attenuate any possible disturbances produced when the water discharges into the vessel. The gas stream was injected coaxially to the water jet with a hypodermic needle whose length, $l$, and inner and outer radii, $r_{i}$ and $r_{o}$ respectively, were varied depending on the experimental conditions (see Table I). The water flow was supplied from a constant pressure tank, and controlled with a flow-meter provided with a high precision valve. The gas flow (either air or helium) was supplied from a pressurized bottle and precisely controlled with a Fisher Bioblock Scientific mass flow-meter. The measurements were performed by recording the images of the coaxial jet with a Photron APX RS high-speed camera
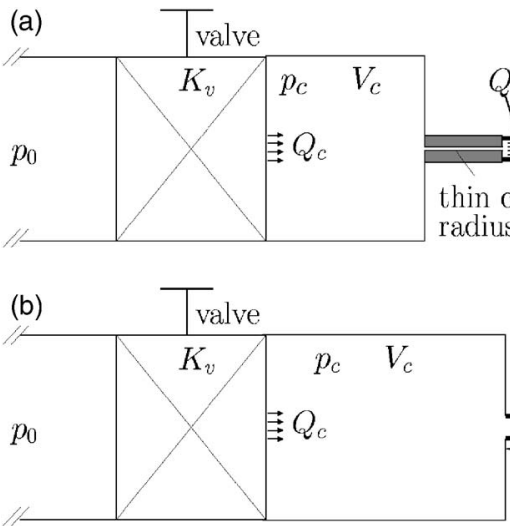
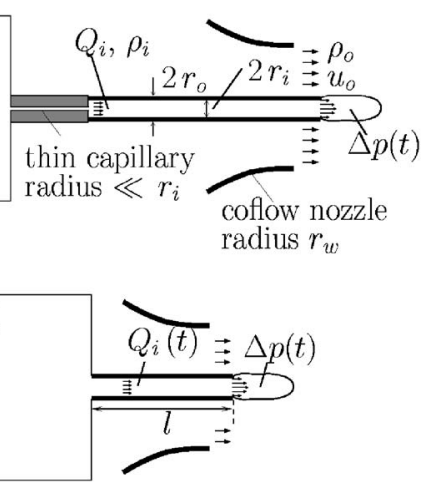

FIG. 3. Detail of the two different experimental setups used in the present work. In (a), constant flow rate conditions are ensured by placing a very thin capillary tube just upstream from the injection needle, while in (b) the gas feeds the needle directly from a chamber of volume $V_{c} \gg l r_{i}^{2}$.

at a rate that varied from 30000 f.p.s., with a resolution of $256 \times 256$ pixels, to 50000 f.p.s. with a $256 \times 128$ pixel resolution.

There are many studies in the literature which show that the gas feeding system may affect the formation of bubbles either through the pressure drop across the injection needle, ${ }^{19}$ or due to acoustic effects. ${ }^{25}$ One of the purposes of the present work is to analyze the effect of the injection conditions on the bubble formation process. Such effect will be elucidated by using the two different experimental configurations, hereafter referred to as setups 1 and 2, sketched in Figs. 3(a) and 3(b), respectively. As illustrated in Fig. 3, the main difference between both configurations is that in setup 1 the gas stream flows through a very thin capillary tube before heading the injection needle. The pressure drop along the capillary tube is much larger than the pressure fluctuation at the needle exit, $\Delta p(t)$, ensuring a constant gas flow-rate feeding the bubble, $Q_{i}$, during the bubble formation process. Note that one needs to be very careful to prevent the presence of void regions, of volume comparable to that of the forming bubble, in the couplings with the capillary tube to guarantee the constant flow-rate conditions. However, in

TABLE I. Geometrical properties of the different needles used in the experiments. Here, $r_{i}$ and $r_{o}$ are the inner and outer radius of the air injection needle, while $l$ denotes its length. The numbers in parentheses in the last column indicate the equation used to estimate $\Delta Q_{i} / Q_{i}$. The use of Eq. (19) to estimate the value of $\Delta Q_{i} / Q_{i}$ in setups of type 2 is justified in view of the values of St and $\mathrm{St} l_{e} / l$ are $\sim \mathcal{O}(1)$.

\begin{tabular}{|c|c|c|c|c|c|c|c|c|}
\hline Expt. & Setup & $\begin{array}{c}r_{i} \\
(\mathrm{~mm})\end{array}$ & $\begin{array}{c}r_{o} \\
(\mathrm{~mm})\end{array}$ & $\begin{array}{c}l \\
(\mathrm{~mm})\end{array}$ & $l_{e} / l$ & St & St $l_{e} / l$ & $\Delta Q_{i} / Q_{i}$ \\
\hline I & 1 & 0.600 & 0.750 & 120 & $\cdots$ & $\cdots$ & $\cdots$ & $\ll 1$ [Eq. (6)] \\
\hline II & 1 & 0.400 & 0.600 & 120 & $\cdots$ & $\cdots$ & $\cdots$ & $\ll 1$ [Eq. (6)] \\
\hline III & 1 & 0.250 & 0.400 & 120 & $\cdots$ & $\cdots$ & $\cdots$ & $\ll 1$ [Eq. (6)] \\
\hline IV & 2 & 0.597 & 0.8255 & 245 & $0.17-0.76$ & $4.7-9.5$ & $1.5-5.2$ & $0.04-0.14$ [Eq. (19)] \\
\hline $\mathrm{V}$ & 2 & 0.419 & 0.635 & 240 & $0.11-0.57$ & $3-8.1$ & $0.9-3.5$ & $0.03-0.17$ [Eq. (19)] \\
\hline VI & 2 & 0.419 & 0.635 & 585 & $0.047-0.215$ & $7.5-20$ & $0.8-3.2$ & $0.015-0.08$ [Eq. (19)] \\
\hline VII & 2 & 0.292 & 0.451 & 215 & $0.14-0.47$ & $3.96-7.65$ & $0.9-2.73$ & $0.05-0.17[$ Eq. (19)] \\
\hline VIII & 2 & 0.597 & 0.8255 & 200 & $0.55-1.77$ & $1.8-5.2$ & $2.5-4.4$ & $0.015-0.032$ [Eq. (19)] \\
\hline IX & 2 & 0.419 & 0.635 & 200 & $0.37-1.77$ & $2.5-4.3$ & $3.3-6.3$ & $0.028-0.059$ [Eq. (19)] \\
\hline $\mathrm{X}$ & 2 & 0.400 & 0.600 & 17 & $1.60-3.00$ & $0.3-0.5$ & $0.75-0.97$ & $0.9-1.2$ [Eq. (19)] \\
\hline
\end{tabular}


setup 2 the injection needle is directly connected to a chamber whose volume $V_{c}$ is much larger than the volume of the needle, $V_{c} \gg \pi r_{i}^{2} l$. As a consequence, depending on the needle geometry, $Q_{i}$ may vary during the bubble formation period as described later on.

At this point it is important to determine the conditions required to guarantee that both, the gas flow-rate measured by the flow-meter, $Q_{c}$, and the pressure in the feeding chamber, $p_{c}$, remain constant during the bubble formation process. Thus, to estimate the relative variation of the gas flow-rate downstream of the control valve, $\Delta Q_{c} / Q_{c}$, we will consider that the upper limit of the pressure fluctuations in the gas reservoir is of the order of the pressure fluctuations within the forming bubble, $\left.\Delta p_{c}\right|_{\max } \sim \Delta p$. Furthermore, a $\mathcal{O}(\Delta p)$ perturbation of the pressure drop across the valve causes a flow-rate variation, $\Delta Q_{c}$, given by

$$
\Delta p(t) \sim K_{v} \rho_{i} Q_{c} \Delta Q_{c} \Rightarrow \frac{\Delta Q_{c}}{Q_{c}} \sim \frac{\Delta p(t)}{p_{0}-p_{c}} \ll 1,
$$

where $\rho_{i}$ is the gas density and $K_{v}$ is the head loss coefficient of the control valve, that indicates that, since in both setups $p_{0}-p_{c} \gg \Delta p$, it can be ensured that $Q_{c}$ remains nearly constant during the bubbling process. Moreover the pressure fluctuations in the feeding chamber, $\Delta p_{c}$, can be estimated using the energy conservation equation as

$$
\frac{\Delta p_{c}}{p_{c}} \sim \frac{\left(Q_{c}-Q_{i}\right) t_{b}}{V_{c}},
$$

with $t_{b}$ being the characteristic bubbling time. In all our experiments, and keeping in mind that typically $t_{b}$ $\sim \mathcal{O}\left(10^{-3} \mathrm{~s}\right), V_{c}$ was chosen to be sufficiently large for the ratio given by Eq. (2) to be very small, even in the extreme case where $Q_{i}-Q_{c}$ was $\mathcal{O}\left(Q_{i}\right)$. Consequently, the condition $\Delta p_{c} / p_{c} \ll 1$ was also accomplished in all the experiments performed, thus ensuring nearly constant pressure conditions at the gas chamber during a bubbling period.

Let us now proceed with the estimation of the pressure fluctuations inside the bubble, $\Delta p(t)$. Using the radial component of the momentum equation, $\Delta p$ can be estimated as ${ }^{39}$

$$
\Delta p \sim \rho_{o} \frac{\partial v_{o}}{\partial t} r_{o} \sim \rho_{o} \frac{v_{o}}{t_{b}} r_{o},
$$

where $\rho_{o}$ is the density of the outer fluid and $v_{o}=\partial r / \partial t$ is the radial velocity at the gas-liquid interface. Moreover, the radial velocity can be estimated, applying the continuity equation to the gas stream, as

$$
2 \pi r_{o} l_{i} v_{o}+\pi r_{o}^{2} u_{o}=Q_{i} \rightarrow v_{o} \sim \frac{r_{o} u_{o}}{2 l_{i}}(U-1),
$$

where $U=Q_{i} /\left(\pi r_{o}^{2} u_{o}\right)$ is the gas-to-liquid mean velocity ratio and $l_{i}$ is the length of the gas ligament attached to the needle exit when the previous bubble has just detached (see Fig. 1). Notice that in Eq. (4) we have taken into account that the axial elongation rate of the bubble is approximately given by the liquid velocity, $u_{o}$. Consequently, $\Delta p$ can be estimated as

$$
\Delta p \sim \frac{\rho_{o}}{t_{b}} \frac{r_{o}^{2} u_{o}}{2 l_{i}}(U-1)
$$

and, for typical values of $t_{b} \sim 10^{-3} \mathrm{~s}, Q_{i} /\left(\pi r_{i}^{2}\right) \sim 10 \mathrm{~m} \mathrm{~s}^{-1}$, $u_{o} \sim 1 \mathrm{~m} \mathrm{~s}^{-1}, l_{i} / r_{o} \sim \mathcal{O}(10)$ and the values of $r_{i}$ and $r_{o}$ provided in Table I, we find that $\Delta p \sim 10^{3} \mathrm{~Pa}$.

Although the flow-rate measured by the flow-meter, $Q_{c}$, and the pressure in the chamber, $p_{c}$, can be considered constant, the gas flow-rate feeding the bubble, $Q_{i}$, may vary with time depending on the experimental conditions. However, in setup $1, \Delta Q_{i} / Q_{i}$, can be estimated as

$$
\frac{\Delta Q_{i}}{Q_{i}} \sim \frac{\Delta p}{p_{c}-p_{a}},
$$

where $p_{a}$ is the ambient pressure. Moreover, since the capillary tube was selected to introduce a pressure drop such that $p_{c}-p_{a} \gg \Delta p$, we can conclude that $\Delta Q_{i} / Q_{i} \ll 1$, indicating that $Q_{i}$ remain nearly constant during the bubbling period in all the experiments performed using setup 1 .

Regarding the experiments performed with setup 2, the gas flow-rate feeding the bubble, $Q_{i}$, may vary during the bubble formation process depending on the geometry of the injection needle. ${ }^{19}$ Thus, to estimate $\Delta Q_{i} / Q_{i}$ as a function of the control parameters, let us first discriminate between the following different cases: (a) cases where the entrance length, defined here as $l_{e}=0.1 Q_{i} / \nu_{i}$, where $\nu_{i}$ is the kinematic viscosity of the gas, and is such that $l_{e} / l \lesssim \mathcal{O}(1)$ and, (b) cases where $l_{e} / l \gg 1$. In the former case, the pressure drop along the needle can be estimated by

$$
\Delta p_{n} \sim 8 \mu_{i} l Q_{i} /\left(\pi^{2} r_{i}^{4}\right),
$$

where $\mu_{i}$ is the gas viscosity. On the other hand, if $l_{e} / l \gg 1$, the pressure drop along the needle can be estimated as

$$
\Delta p_{n} \sim \frac{1}{2} \rho_{i} \frac{Q_{i}^{2}}{\pi^{2} r_{i}^{4}} .
$$

The gas flow-rate feeding the bubble, $Q_{i}$, will be constant if

$$
\frac{\Delta p}{\Delta p_{n}} \sim \frac{\Delta Q_{i}}{Q_{i}} \ll 1,
$$

with $\Delta p_{n}$ given by Eqs. (7) and (8) depending on each particular case. At this point, note that Eq. (9) is a sufficient, but not necessary condition to ensure a constant flow-rate at the exit of the injection needle. Indeed, the additional pressure drop along the needle caused by the unsteady gas acceleration, $\Delta p_{u}=l /\left(\pi r_{i}^{2}\right) \rho_{i} \partial Q_{i} / \partial t$, can be dominant over $\Delta p_{n}$ for sufficiently small values of the bubble break-up time, $t_{b}$. Thus, to properly estimate $\Delta Q_{i} / Q_{i}$, it is convenient to determine the dominant mechanism responsible for the pressure drop along the injection needle. For this purpose, let us consider the following two different situations that arise when comparing the unsteady term, $\Delta p_{u}$, with either expressions (7) and (8):

(1) If $l_{e} / l \gg 1$, 


$$
\frac{\Delta p_{u}}{\Delta p_{n}} \sim \frac{l \pi r_{i}^{2}}{t_{b} Q_{i}}=\mathrm{St},
$$

where the Strouhal number, St, can also be understood as the needle-to-bubble volume ratio.

(2) If $l_{e} / l \lesssim \mathcal{O}(1)$,

$$
\frac{\Delta p_{u}}{\Delta p_{n}} \sim \mathrm{St} \frac{l_{e}}{l} .
$$

Consequently, if $\mathrm{St} \geqslant \mathcal{O}(1)$ when $l_{e} / l \gg 1$ or $\mathrm{St} l_{e} / l$ $\gtrsim \mathcal{O}(1)$ when $l_{e} / l \leqq \mathcal{O}(1), \Delta Q_{i} / Q_{i}$ must be estimated through the balance

$$
\Delta p_{u} \sim \rho_{i} l \frac{\Delta Q_{i}}{t_{b} \pi r_{i}^{2}} \sim \Delta p(t),
$$

which leads to

$$
\frac{\Delta Q_{i}}{Q_{i}} \sim \frac{\Delta p t_{b} \pi r_{i}^{2}}{Q_{i} \rho_{i} l} \sim \frac{\rho_{o}}{\rho_{i}} \frac{r_{i}^{2}}{2 l_{i} l}\left(1-\frac{1}{U}\right),
$$

with $\Delta p$ estimated from Eq. (5). Furthermore, Sevilla et al. ${ }^{23}$ demonstrated that the condition $\left(1-\pi r_{o}^{2} u_{o} / Q_{i}\right) \sim \mathcal{O}(1)$ is necessary to have a bubbling regime rather than a jetting regime and, consequently, Eq. (13) may be further simplified to

$$
\frac{\Delta Q_{i}}{Q_{i}} \sim \frac{\rho_{o}}{\rho_{i}} \frac{r_{i}^{2}}{2 l_{i} l} .
$$

The estimate of $\Delta Q_{i} / Q_{i}$ given by (14) should be modified if $c t_{b}<l$, where $c$ is the sound speed in the gas, since, in such case, the pressure perturbation at the needle exit would be $\Delta p_{u} \sim \rho_{i} \Delta Q_{i} c / \pi r_{i}^{2}$. Consequently, $l$ in Eq. (13) must be replaced by $c t_{b}$. Nevertheless, this discussion might be only relevant for the longest needles used in our experiments $(l$ $\gtrsim 2 \times 10^{-1} \mathrm{~m}$ ). However, even in those cases the length of the injection needle was of the order of $c t_{b}$ and $l$ does not need to be substituted by $c t_{b}$.

In view of the above discussion, the conditions that guarantee a constant gas flow-rate entering the bubble in setup 1 are

$$
\frac{\Delta p}{\Delta p_{v}} \ll 1, \quad \frac{Q_{i} t_{b}}{V_{c}} \ll 1,
$$

together with a pressure drop along the capillary tube, $p_{c}$ $-p_{a}$, much larger than the pressure fluctuation at the needle exit, $\Delta p(t)$. Here $\Delta p$ can be estimated through Eq. (5) and $\Delta p_{v} \sim K_{v} \rho_{i} Q_{c}^{2}$ represents the pressure drop across the control valve. However $Q_{i}$ will remain constant during the breakup time when a configuration of type 2 is used if the conditions in Eq. (15) are satisfied, together with the following additional conditions:

(1) If $l_{e} / l \gg 1$ and $\mathrm{St} \ll 1$,

$$
\frac{\Delta p}{\Delta p_{n}} \ll 1,
$$

with $\Delta p_{n}$ given by Eq. (8).

(2) If $l_{e} / l \gg 1$ and St $\gtrsim \mathcal{O}(1)$

$$
\frac{\rho_{o}}{\rho_{i}} \frac{r_{i}^{2}}{2 l_{i} l} \ll 1
$$

(3) If $l_{e} / l \lesssim \mathcal{O}(1)$ and St $l_{e} / l \ll 1$

$$
\frac{\Delta p}{\Delta p_{n}} \ll 1,
$$

with $\Delta p_{n}$ given by Eq. (7).

(4) Finally, if $l_{e} / l \lesssim \mathcal{O}(1)$ and St $l_{e} / l \gtrsim \mathcal{O}(1)$,

$$
\frac{\rho_{o}}{\rho_{i}} \frac{r_{i}^{2}}{2 l_{i} l} \ll 1
$$

A large number of experiments, with gas and liquid velocities within the ranges $3 \mathrm{~m} / \mathrm{s}<Q_{i} /\left(\pi r_{i}^{2}\right)<30 \mathrm{~m} / \mathrm{s}$ and $1 \mathrm{~m} / \mathrm{s}<u_{o}<3 \mathrm{~m} / \mathrm{s}$, respectively, and gas Reynolds number $\operatorname{Re}_{i}=Q_{i} /\left(\pi \nu_{i} r_{i}\right)<2300$ (laminar pipe flow), were performed with injection needles of different length. Note also that the liquid jet discharges directly into a stagnant air atmosphere and, consequently, buoyancy effects are negligible in all the experiments presented here and that the gas-to-liquid mean velocity ratio was larger than unity $\left[Q_{i} /\left(\pi r_{o}^{2} u_{o}\right)>1\right]$ to ensure a bubbling regime instead of a jetting one ${ }^{23}$ A summary of the experimental conditions of each experiment is provided in Table I, with the type of experimental setup employed indicated in the second column. In this table the experimental sets IV-VII correspond to experiments reported in Sevilla et ${ }^{39}{ }^{39}$

It is important to indicate that the experiments performed with both type of experimental setups satisfied the conditions given by Eqs. (1) and (2) and, therefore, the measured flowrate injected into the chamber, $Q_{c}$, and the chamber pressure, $p_{c}$, were nearly constant during a bubbling period. Furthermore, as shown in Table I, the only experiments for which the flow rate entering the bubble is strictly constant $\left(\Delta Q_{i} / Q_{i} \ll 1\right)$ are those performed with setup 1 (data sets I-III). However, $Q_{i}$ can also be considered nearly constant for the experiments performed with setup type 2 and long injection needles (data sets IV-IX). On the other hand, $Q_{i}$ varies during the bubbling time for data set $\mathrm{X}$ since $\Delta Q_{i} / Q_{i} \sim \mathcal{O}(1)$. Moreover, it will be shown in Sec. III that the differences in the bubbling frequency obtained in several experiments performed for the same values of $u_{o}, Q_{c}, r_{i}$, and $r_{o}$ but different needle lengths, rely on the distinct injection conditions.

\section{ANALYSIS OF RESULTS}

This section is devoted to the presentation and analysis of the experimental results obtained using the two experimental setups described in Sec. II. Moreover, we will provide the correct scaling for both the bubbling frequency and the bubble size as functions of the different control parameters. In order to gain more insight into the physics of the phenomenon, a detailed comparison with corresponding numerical results will also be reported and discussed. 
(a)
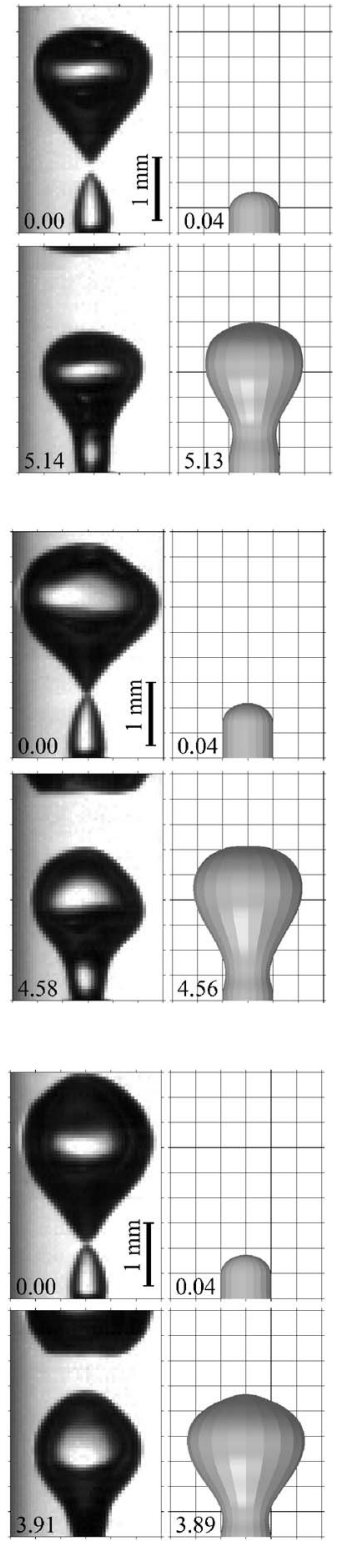

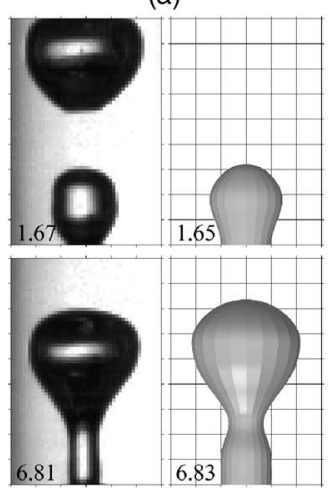

(b)
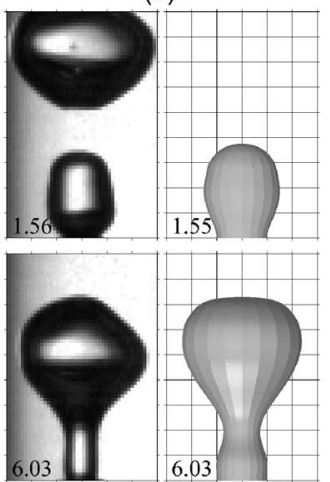

(c)

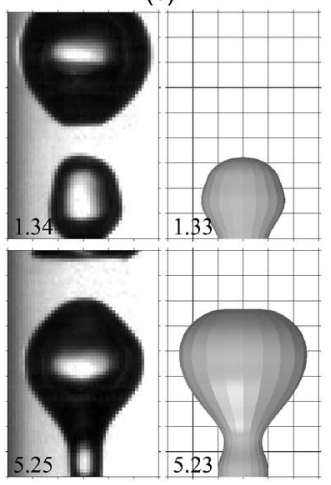

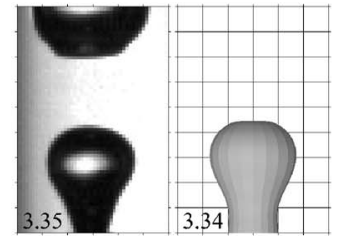
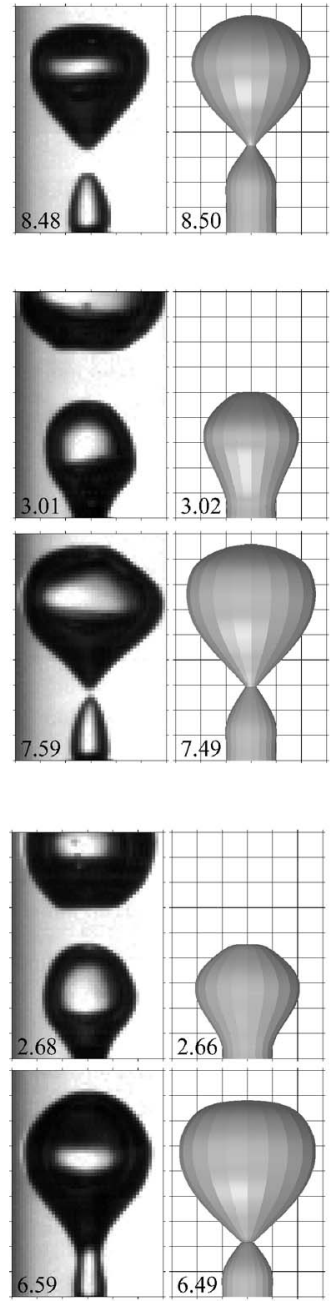

FIG. 4. Comparison of the experimental visualizations with the numerical simulations at $\mathrm{We}_{o}=10.24$. (a) $U$ $=1.86$, (b) $U=2.60$, and (c) $U=4.98$. The experimental conditions are those corresponding to experimental set III reported in Table I.

\section{A. Constant flow rate injection conditions}

In this subsection we will compare the results of experiments performed with setup 1 at constant flow rate conditions with the numerical results obtained from the simulations performed with the boundary integral method (BIM) described in the Appendix. Although both the nonlinear evolution of the bubble interface and the coupling with the injection needle are taken into account in our approach, we can anticipate that there will be some differences between the numerics and the experiments. The discrepancies can be partly attributed to the fact that unlike what experimentally happens, the BIM considers an infinitely thin gas-water shear layer. Moreover, in the simulations we assumed that the gas is injected through a needle of zero wall thickness and radius $r_{o}$. However our experiments revealed that, after exiting the needle through the inner radius, $r_{i}$, the radius of the gas interface expands to a value close to $r_{o}$ a few radii downstream of the needle exit. Although the ratio $\left(1-r_{i} / r_{o}\right)$ $\simeq 0.3$ is small, we observed that, keeping the rest of the parameters constant, the bubble formation frequency decreased as the needle thickness increased.

Figures 4-6 compare the numerically calculated time evolution of the bubble interface with that measured experimentally for different values of the Weber number, defined here as $\mathrm{We}_{o}=\rho_{o} u_{o}^{2} r_{o} / \sigma$ with $\sigma$ being the surface tension, and the gas-to-liquid mean velocity ratio, $U=Q_{i} /\left(\pi r_{o}^{2} u_{o}\right)$. The images shown in Fig. 4 were taken using the experimental set III of Table I, while those of Figs. 5 and 6 correspond to results obtained with the experimental set II. The figures illustrate the differences on the bubble shape at detachment with the Weber number. In addition, Figs. 4-6 also indicate that the bubbling time decreases when $U$ increases for a fixed value of $\mathrm{We}_{o}$, while it increases with $\mathrm{We}_{o}$ for a fixed value of $U$ [compare, for instance, Figs. 5(a) and 6(a)]. Consequently, both the difference of velocity between the gas and the liquid streams, and the surface tension forces contribute to acceler- 

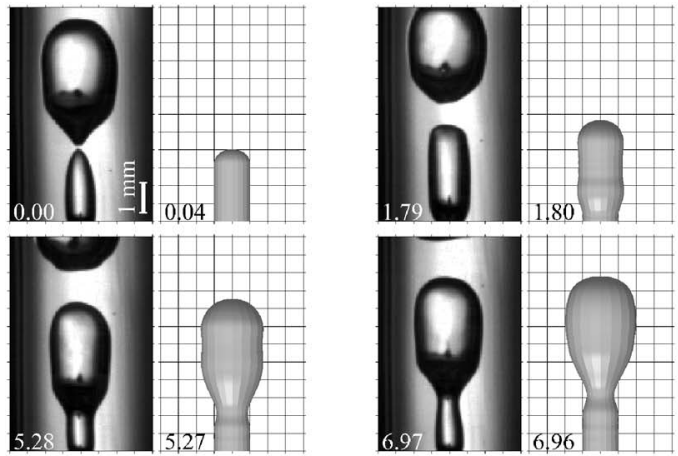

(b)
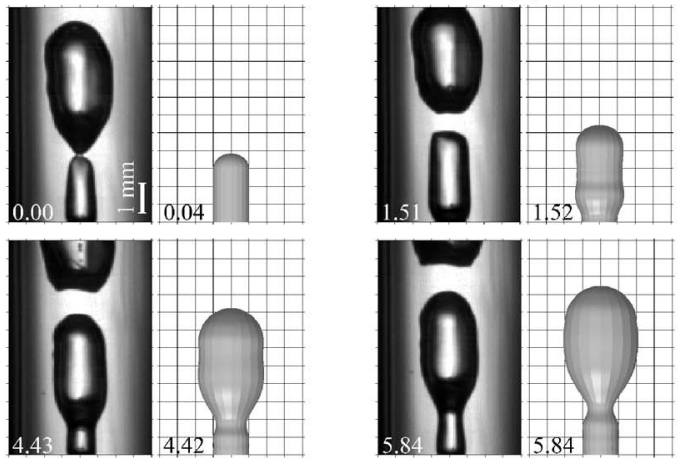

(c)
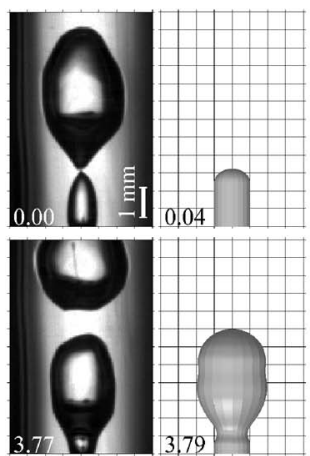
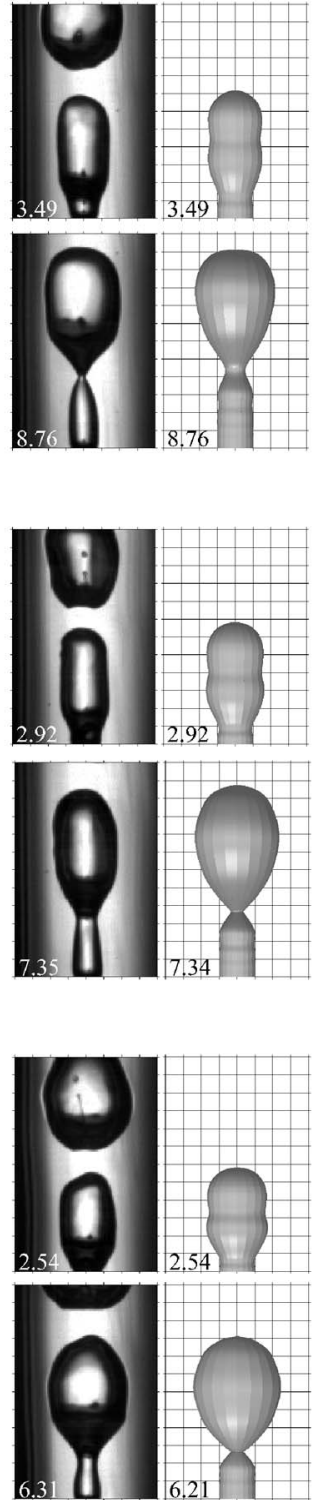

FIG. 5. Comparison of the experimental visualizations with the numerical simulations at $\mathrm{We}_{o}=24.61$. (a) $U$ $=3.05$, (b) $U=3.94$, and (c) $U=4.82$. The experimental conditions are those corresponding to experimental set II reported in Table I. ate the bubble formation process. It is also interesting to note that the presence of the liquid co-flow prevents bubble coalescence even for values of $U$ as large as 10 .

To guarantee that the gas flow rate in setup 1 was indeed constant during the bubbling process we measured the time evolution of the bubble volume from the high speed movies recorded. The result of this analysis has been displayed in Fig. 7, where the dimensionless bubble volume obtained from the images, $V_{b}=\int_{0}^{z_{t}} \pi r_{s}^{2}(z) d z /\left(\pi r_{o}^{3}\right)$, has been plotted as a function of dimensionless time, $\tau=t u_{o} / r_{o}$, for four different cases, namely $\mathrm{We}_{o}=10.24, U=1.86$ (a), $\mathrm{We}_{o}=15.75, U$ $=3.84$ (b), $\mathrm{We}_{o}=24.61, U=4.82$ (c), and $\mathrm{We}_{o}=48.23, U$ (a)
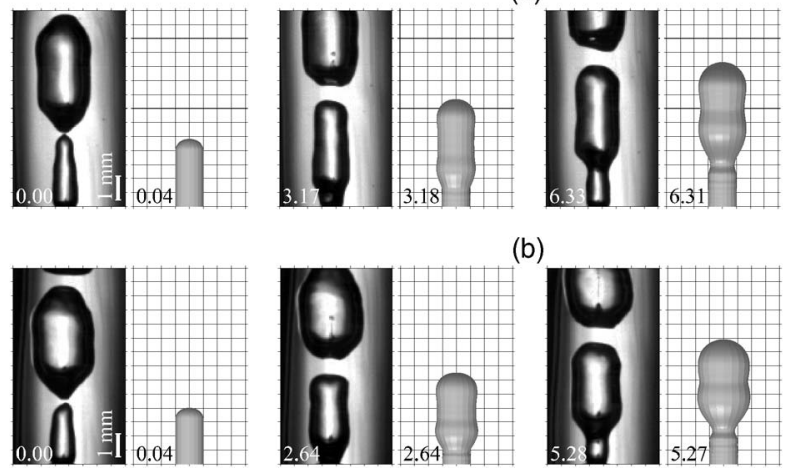

(b)

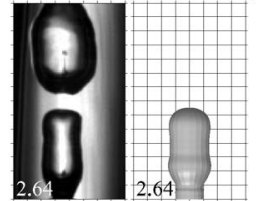

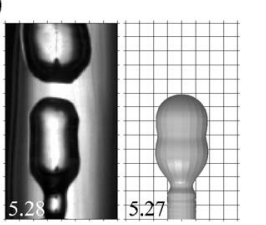
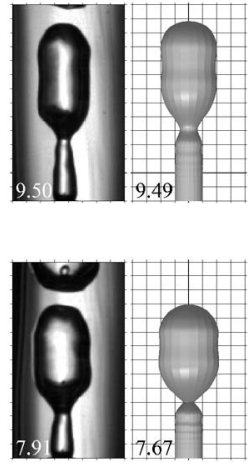

FIG. 6. Comparison of the experimental visualizations with the numerical simulations at $\mathrm{We}_{o}=48.23$. (a) $U$ $=3.09$, (b) $U=3.77$. The experimental conditions are those corresponding to experimental set II reported in Table I. 

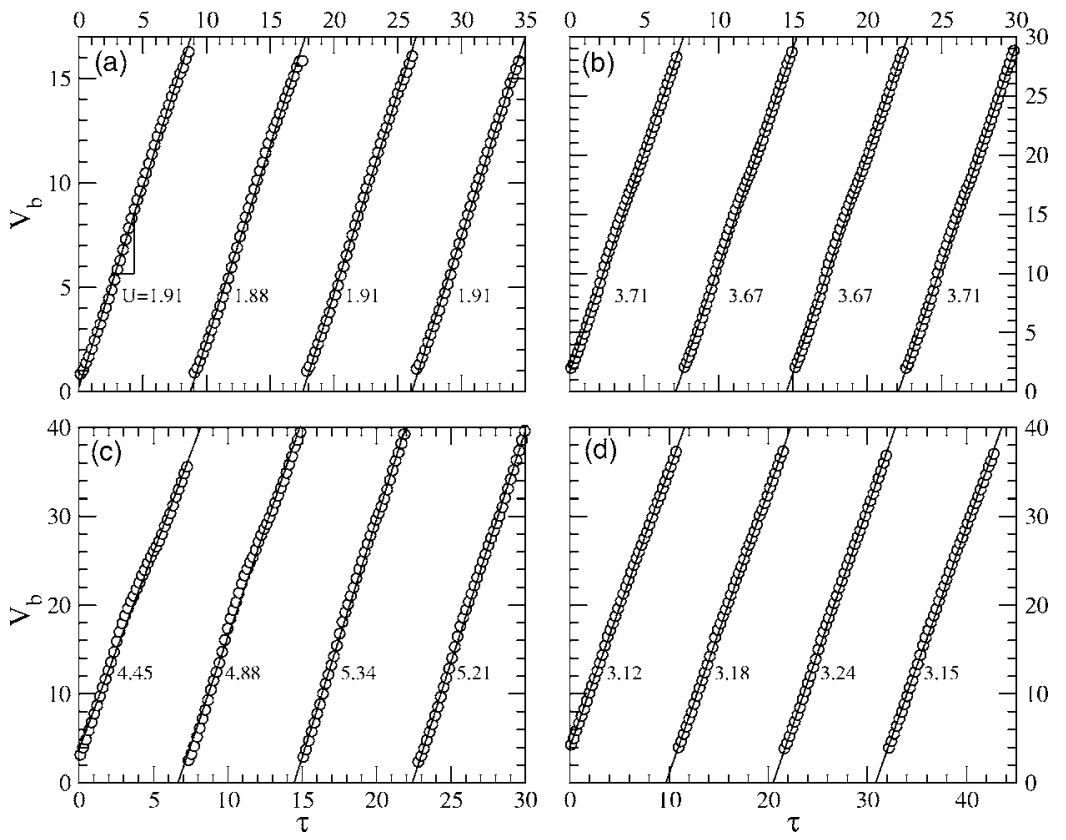

FIG. 7. Time evolution of the volume of the bubble. (a) $\mathrm{We}_{o}=10.24, U=1.86$, (b) $\mathrm{We}_{o}=15.75, U=3.84$, (c) $\mathrm{We}_{o}=24.61, U=4.82$, and (d) $\mathrm{We}_{o}=48.23, U=3.09$. Symbols indicate the experimental data and the solid line represents a linear fit. $=3.09(\mathrm{~d})$. Here, $r_{s}(z)$ represents the radius of the interface obtained from the image, $z$ is the axial coordinate, and $z_{t}$ denotes the bubble tip, defined as $r_{s}\left(z_{t}\right)=0$. Note that, with this notation, a strictly constant flow rate implies the simple bubble growth law $V_{b}=V_{0}+U \tau$, where $V_{0}$ is the initial bubble volume and the slope corresponds to the velocity ratio parameter, $U$. The symbols in Fig. 7 indicate the experimental data points, the solid lines are a linear fit for each bubbling event, and the numbers are the corresponding slopes. Notice the linearity appreciated in all the bubbling events (the correlation coefficient was larger than 0.99 in all cases) and the close agreement between the values of the slopes and the corresponding nominal values of $U$. These results support our premise that the setup 1 guarantees constant flow rate conditions, as will be assumed in the remainder of the paper. At this point we would like to emphasize that the only entries for the numerical simulation are the experimentally measured values of $\mathrm{We}_{o}$ and $U$, as well as the initial length of the gas stem attached to the needle exit, $l_{i}$. Since the dimensionless time, $\tau$, is the same in both, the experiments and the simulations, the agreement observed in Figs. 4-6 is obtained without any free adjustable parameter. Moreover, we did not take into account the interaction between neighboring bubbles, whose influence has thus proven not to be essential, in order to properly describe a bubbling period within the range of parameters under study here.

Making use of the numerical simulations, we examined the mechanisms leading to the bubble growth and subsequent break-up in detail. First, to analyze the effect of the gas inertia on the bubble formation process, we compared the high-speed visualizations with the numerical simulations performed including the gas convective terms as well as neglecting them. Notice that, to perform the simulations neglecting the gas inertia, as described in the Appendix, the Bernoulli equation (A8) was used instead of Eq. (A6). The results of these comparisons are displayed in Fig. 8, where the simulations without including gas inertia, Fig. 8(b), follow the observed experimental behavior except during the final instants previous to pinch-off. In effect, note that in the last frame of Fig. 8(b), $\tau \approx 8.9$, the neck of the gas stem is still collapsing in the numerical simulations while the bubble has already detached from the needle in the experimental visualizations. On the other hand, as observed in Fig. 8(a), the whole process seems to be well captured if gas inertia is included; the neck forms at $\tau_{\exp } \approx 4.9$ and the bubble pinches off at $\tau_{b} \approx 7.3$ in both the numerical simulations and in the (a)
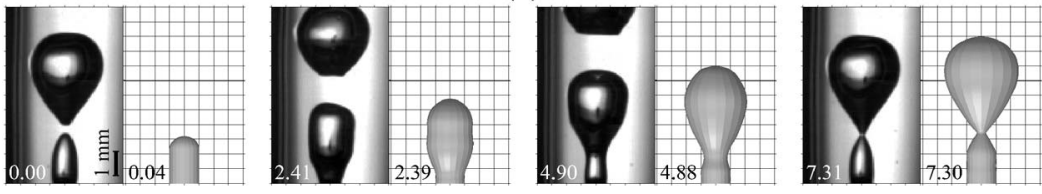

(b)
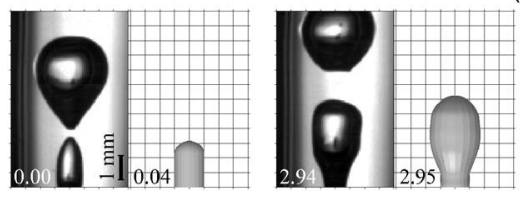

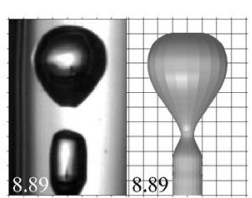

FIG. 8. Comparison of the experimental visualizations with the numerical simulations at $\mathrm{We}_{o}=15.75$ and $U$ $=3.84$ : (a) with gas inertia, and (b) without including gas inertia. The experimental conditions are those corresponding to experimental set II reported in Table I. 

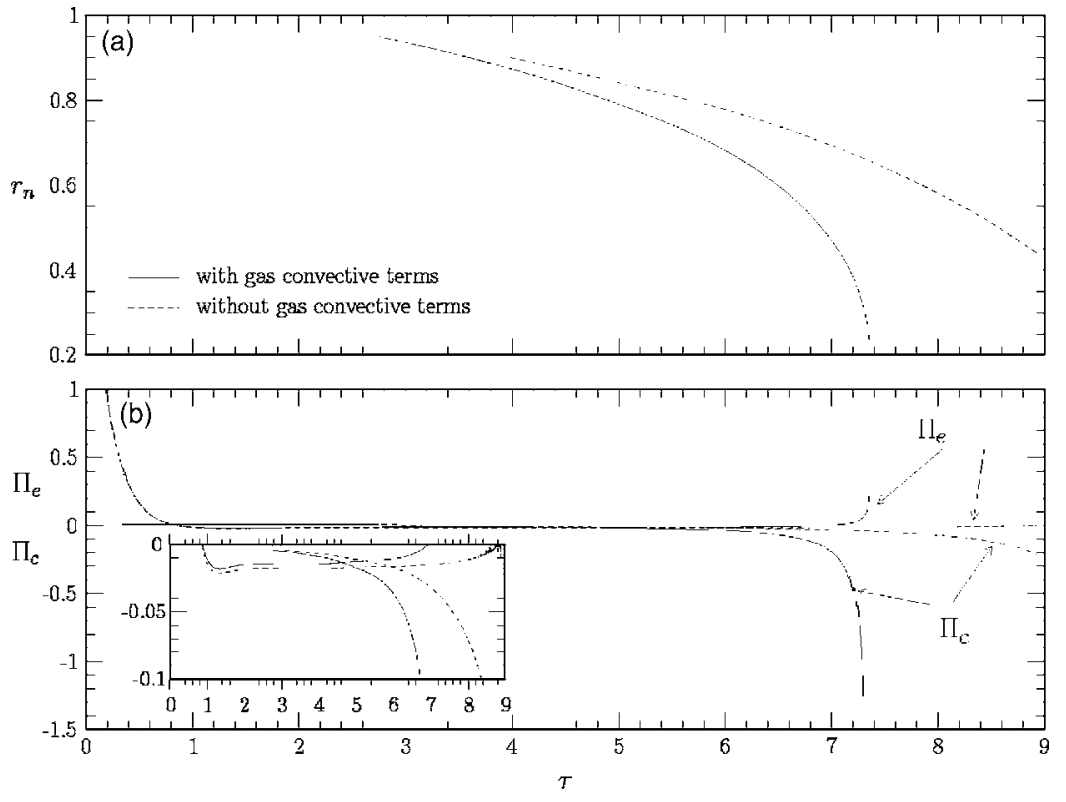

FIG. 9. (a) Time evolution of the radius of the neck including gas inertia (solid line) and without including gas inertia (dashed line). (b) Time evolution of the pressure at the needle exit, $\Pi_{e}$, and $\Pi_{c}=1 / 2 \rho_{i} / \rho_{o}\left(U^{2}-u_{n}^{2}\right)$ including gas inertia (solid line) and without gas inertia (dashed line). Here $u_{n}$ is the dimensionless gas velocity at the neck of the gas ligament, $\mathrm{We}_{o}=15.75$ and $U$ $=3.84$. corresponding experiment. This result indicates that the pressure drop along the minimum radius, $r_{n}$, promoted by the gas convective acceleration (the Venturi effect already described in Sevilla et al. ${ }^{39}$ and Gordillo et al. ${ }^{11}$ ), is not relevant to describe the initial time evolution of the bubble interface, although it is essential to describe the latest stages of the breakup process. Moreover, the Venturi effect also explains the discrepancy between the slender, symmetric evolution of the neck provided by the numerical simulations which do not include gas inertia $^{11,12}$ [Fig. 8(b)] and the nonslender, asymmetric evolution of the neck observed at the final instants of pinch-off ${ }^{11}$ [Fig. 8(a)].

The role of gas inertia can be appreciated more clearly in Fig. 9(a), where we have compared the time evolution of the radius of the neck given by our simulations, including and without including the gas inertia, at $\mathrm{We}_{o}=15.75$ and $U$ $=3.84$. Notice the rapid collapse of the neck, caused by the Venturi effect, in the simulations that contemplate the gas inertia. Moreover, the time evolution of the pressure at the needle exit, $\Pi_{e}=-\rho_{i} / \rho_{o} \partial \phi_{i} / \partial \tau$, and the contribution of the convective terms to the pressure drop along the minimum radius, $\Pi_{c}=1 / 2 \rho_{i} / \rho_{o}\left(U^{2}-u_{n}^{2}\right)$ are also plotted in Fig. $9(\mathrm{~b})$ for both type of simulations with $u_{n}$ being the dimensionless gas velocity at the neck of the gas ligament of radius $r_{n}$. It can be observed in the inset of Fig. 9(b) that $\Pi_{c}$ is the dominant contribution for $\tau>\tau^{*} \approx 5$, indicating that $\Pi_{c}$ is the pressure term that controls the final instants of bubble break-up. As explained in Gordillo et al., ${ }^{11}$ the large negative pressure drop at $r_{n}$ is a consequence of the fact that the gas flow rate through the minimum radius remains constant during the pinch-off process, $Q_{i} /\left(\pi r_{i}^{2} u_{o}\right) \simeq r_{n}^{2} u_{n}$, which on physical grounds, can be explained as follows. Far from the neck, the radial liquid velocity at the bubble interface does not change appreciably during times close to pinch-off. Thus, the rate of change of the bubble volume is nearly constant during pinchoff and, consequently, there is a constant flow-rate of gas flowing through the neck which moves downstream with an axial velocity approximately equal to the liquid velocity and, thus, much smaller than $u_{n}, u_{o} \ll u_{n}$.

In order to provide additional evidence of the relevance of gas inertia in the description of the final instants of bubble break-up at constant flow-rate injection conditions, we carried out a series of experiments with the geometrical configuration reported in set II of Table I, but using helium as a working gas instead of air, under the same conditions of $Q_{i}$, $u_{o}$, and $\rho_{o}$ of previous experiments performed with air ( $\rho_{\text {air }} / \rho_{\mathrm{He}} \simeq 6.72$ ). It should be noticed that changes in the nature of gas employed only affects to the gas density, modifying by less than $0.1 \%$ the gas-water surface tension coefficient. ${ }^{40}$ Figure 10 shows two bubbling periods corresponding to experiments performed with helium [Fig. 10(a)], and air [Fig. 10(b)] for $\mathrm{We}_{o}=15.75$ and $U=6$. Since the gas does not appreciably affect the surface tension of gas-liquid interfaces, to obtain the Weber number in the experiments performed with helium, the helium-water surface tension was taken the same as that of air-water $\left(\sigma=0.072 \mathrm{~N} \mathrm{~m}^{-1}\right)$. Although we found that the density of the gas did not significantly affect the bubbling frequencies at constant flow conditions, the geometry of the formed bubbles differs substantially from each other. Indeed, Fig. 10 shows that the top of the helium bubbles is flatter than that of the air bubbles. In addition, it can also be observed that the surface of the helium bubbles is smoother than the surface of the air bubbles. These differences can be explained in terms of the different inertia of the gas flow through the neck. Note that the pressure inside the gas ligament in the region near the neck decreases as the density of the gas increases and, consequently, the efficiency of the Venturi effect already mentioned before also increases. The negative pressure inside the neck accelerates the liquid flow surrounding it, and produces a liquid jet penetrating into the newly formed bubble at a given velocity once the bubble detaches from the gas stem [observe 
(a)
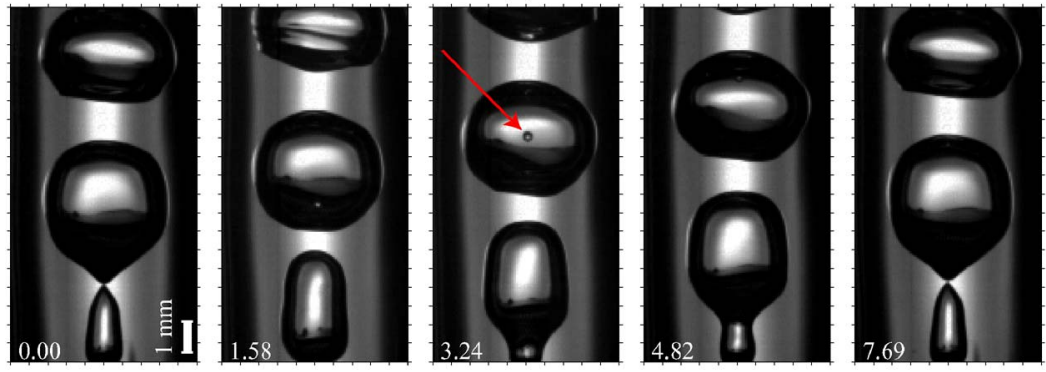

(b)
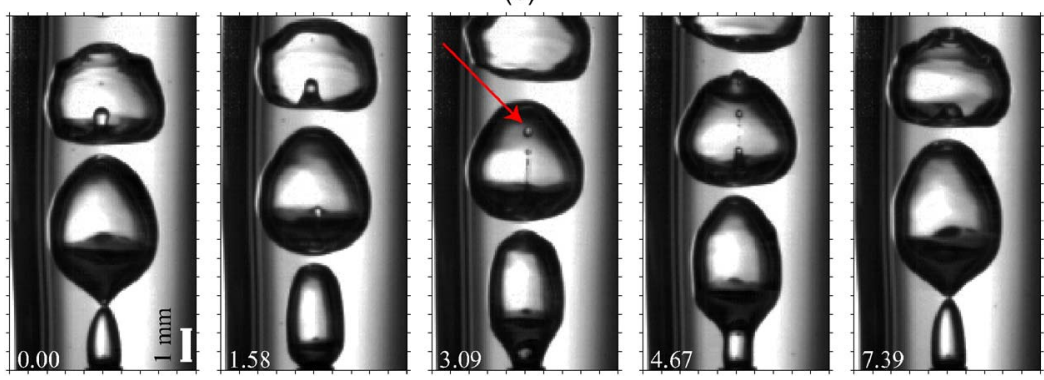

FIG. 10. Time evolution of the bubble formation process at $\mathrm{We}_{o}=15.75$ and $U=6$ for two different gases: (a) helium and (b) air. The experimental conditions are those corresponding to experimental set II reported in Table I. the sequences at $\tau=3.09$ and $\tau=4.67$ of Fig. 10(b)]. To quantify this effect, we considered the singular liquid jets that penetrate into the gas bubbles immediately after their pinchoff, and that are typical of a large number of cavity collapse processes, ${ }^{41}$ and estimated the characteristic axial velocity of the water jet entering the gas bubble by measuring the position of the water drop marked by an arrow in Fig. 10 at two different times. The analysis provided with a velocity of the water drop inside the air bubble of approximately $5 \mathrm{~m} \mathrm{~s}^{-1}$, whereas the droplet velocity inside the helium bubble was approximately $3 \mathrm{~m} \mathrm{~s}^{-1}$. It seems clear that the velocity of the water jet penetrating the bubble is substantially larger in air than in helium, what leads to the different geometries observed in the photographs of Fig. 10. In fact the frame at $\tau$ $=4.67$ in Fig. 10(b) shows the impact of the inner water jet on the top of the air bubble, identified by a cusp or protuberance on its surface, and not present on the helium bubble. In addition, another evidence that supports the key influence of gas density during the final instants previous to pinch-off is that the gas inertia has also been shown to play an essential role in the generation of satellite bubbles. ${ }^{4}$

After describing what happens in the region near the neck at times close to pinch-off, let us now go back to Fig. 9 to analyze the time evolution of the pressure at the needle exit, $\Pi_{e}$, and its effect on the bubble formation process. Although, it has already been shown that the gas inertia is crucial to describe the final instants of the breakup process, the gas convective acceleration within the bubble is negligible during the initial stages of the process as can be observed by comparing the values of $\Pi_{e}$ and $\Pi_{c}$ from $\tau=0$ to $\tau \simeq 5$ provided by the numerical calculations performed with and without convective terms and shown in Fig. 9(b). This result explains why our numerical simulations performed neglecting the gas convective terms nearly follow the experimental visualizations during most part of the formation process, except at times close the breakup instant [see Fig. 8(b)]. Furthermore, observe in Fig. 9(b) that $\Pi_{e}$ is initially positive and causes an initial outwards radial acceleration of the liquid surrounding the gas stem. However, $\Pi_{e}$ becomes negative at $\tau \simeq 1$, leading to the inwards radial acceleration of the liquid and the subsequent generation of the neck. Consequently, the initial stages of the bubble formation process are dominated by a balance among liquid inertia, surface tension, and the gas pressure at the needle exit. Thus, since the gas convective acceleration within the bubble is not essential to describe the initial formation of the bubble, the duration of the expansion stage, or expansion time, is expected to scale with the liquid convective time, $t_{\exp } \sim r_{o} / u_{o}$, and to depend only on $U$ and $\mathrm{We}_{o}$. In order to check our conjecture, we measured the expansion time, $t_{\text {exp }}$, by processing the highspeed visualizations. To perform the analysis, we decided to define the end of the expansion stage as the moment when the radius of neck was equal to $0.9 r_{g}$, where $r_{g}$ is the radius of the gas ligament upstream from the neck. Each case was averaged over more than 15 bubbling events with the aim at reducing the measurement errors as much as possible. The result of the analysis is displayed in Fig. 11, where the expansion time, made dimensionless with the liquid convective time, $\tau_{\exp }=t_{\exp } u_{o} / r_{o}$, has been plotted as a function of $U$ for several values of $\mathrm{We}_{o}$ for the experimental sets III [Fig. 11(a)] and II [Fig. 11(b)]. The figure shows that, for a fixed value of the Weber number, $\tau_{\exp }$ slightly increases when $U$ decreases, specially for small values of $U$, and that $\tau_{\exp }$ increases with $\mathrm{We}_{o}$, what indicates that both surface tension and gas-water shear tend to accelerate the formation of the neck. Figure 11 also illustrates that the values of $\tau_{\exp }$ obtained in the experimental set III, whose needle has an innerto-outer radius ratio of $r_{i} / r_{o}=0.625$, are slightly larger than those obtained in set II, where $r_{i} / r_{o}=0.667$. Thus, we can infer that the needle thickness does in fact influence the time scale of the expansion stage and the bubbling frequency, such that the bubble formation frequency decreases as the relative needle thickness increases. However, since $\tau_{\text {exp }}$ remains nearly constant at large Weber numbers, and having in 


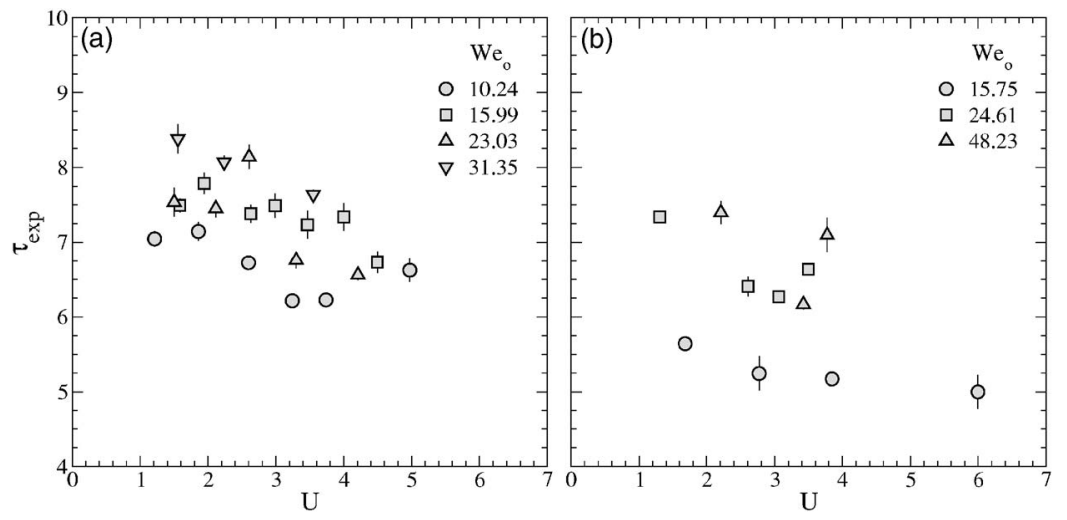

FIG. 11. Dependence of the expansion time, $\tau_{\text {exp }}$, on the gas-to-liquid velocity ratio, $U$, for several values of the Weber number, $\mathrm{We}_{o}$, for (a) experimental set III and, (b) experimental set II. The error bars indicate the standard deviation of the measurements.

mind that it is just a rough approximation, we decided to consider $\tau_{\text {exp }}$ a constant that only varies with the thickness of the injection needle under consideration $\left[\tau_{\exp } \sim k_{1}\left(r_{i} / r_{o}\right)\right.$, for needles III and II, respectively].

Moreover, the bubbling time arises as the addition of the expansion time plus the collapse time. As already discussed above, the relevant time scale of the collapse stage can be estimated by noting that the inward acceleration of the gas ligament is produced by a favorable pressure gradient directed towards the neck. In order to provide an estimation of $\Pi_{e}$, which governs the initial instants of the collapse stage, the bubble can be modeled as a cylinder of variable radius $r_{b}(t)$ and constant length $l_{b}$ attached to cylindrical gas stem of radius $r_{o}$ and a length that varies at rate of $d l / d t=u_{o}$, as revealed by the analysis of the experiments. Applying the continuity equation to the forming bubble, one has

$$
\pi \frac{d r_{b}^{2}}{d t} l_{b}+\pi r_{o}^{2} u_{o}=Q_{i} \rightarrow \frac{r_{b}}{r_{o}}=\left(1+(U-1) \frac{r_{o} \tau}{l_{b}}\right)^{1 / 2} .
$$

Therefore the negative pressure accelerating the neck inwards can be estimated from the radial momentum equation as $\Pi_{e} \rho_{o} u_{o}^{2} / r_{o} \sim-\partial p / \partial r \sim \rho_{o} \partial^{2} r_{b} / \partial t^{2}$. Since, as suggested from Fig. 9, the negative gas pressure at the needle exit is the dominant mechanism provoking the initial stages of collapse with $k_{1} \sim 7.5$ for $r_{i} / r_{o}=0.625$ and $k_{1} \sim 6.5$ for $r_{i} / r_{o}=0.667$

process for large values of $U$, the preceding balance yields

$$
\rho_{o} u_{o}^{2} \Pi_{e} \sim \rho_{o} r_{b} \frac{d^{2} r_{b}}{d t^{2}} \sim \rho_{o} r_{n} \frac{d^{2} r_{n}}{d t^{2}} .
$$

Thus, the estimate of $r_{b}$ through Eq. (20) provides the following scaling for the collapse time:

$$
t_{\mathrm{col}} \sim \frac{l_{b}}{u_{o}} \frac{1}{U-1} .
$$

Since the gas ligament attached to the needle exit elongates axially at a velocity $\simeq u_{o}, l_{b}$ in Eq. (22) can be estimated as $l_{b} \simeq l_{i}+u_{o} t_{\exp } \simeq l_{i}+k_{1} r_{o}$ where $k_{1}$ is a constant value. Consequently, estimating $l_{i}$ as $l_{i} / r_{o} \simeq k_{2}-k_{3} U$, with $k_{2}$ and $k_{3}$ also constants of order unity, the collapse time in Eq. (22) yields

$$
\tau_{\mathrm{col}}=\frac{t_{\mathrm{col}} u_{o}}{r_{o}} \simeq \frac{\left(k_{1}+k_{2}\right)-k_{3} U}{U-1}
$$

and the total bubbling time $t_{b}=t_{\mathrm{exp}}+t_{\mathrm{col}}$, can be approximated by

$$
\tau_{b}=\frac{t_{b} u_{o}}{r_{o}} \simeq \frac{\left(k_{1}-k_{3}\right) U+k_{2}}{U-1}=\frac{k^{*} U+k_{2}}{U-1},
$$

with $k^{*}=k_{1}-k_{3}$.

Figure 12 shows the dependence of the bubbling fre-

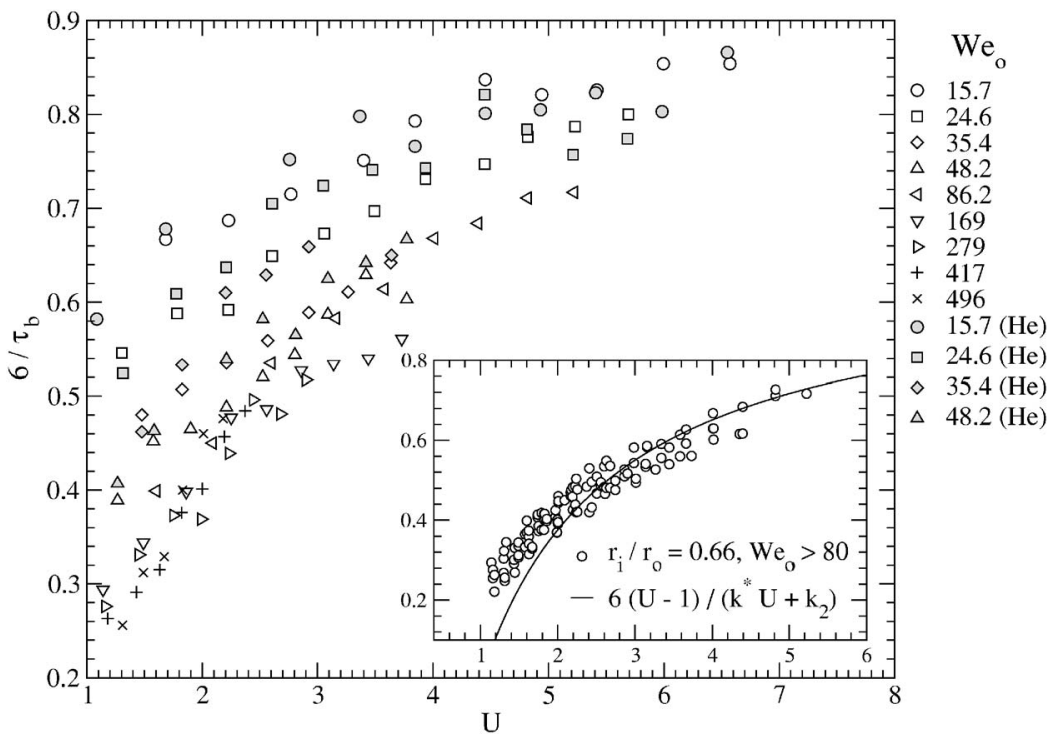

FIG. 12. Bubbling frequencies at constant flow-rate conditions. Hollow symbols correspond to experiments performed with air and solid symbols indicate experiments performed with helium. The inset shows the experimental results obtained at $\mathrm{We}_{o} \gg 1$, together with the fit provided by Eq. (24), where $k^{*}=5.84$ and $k_{2}$ $=4.29$. 


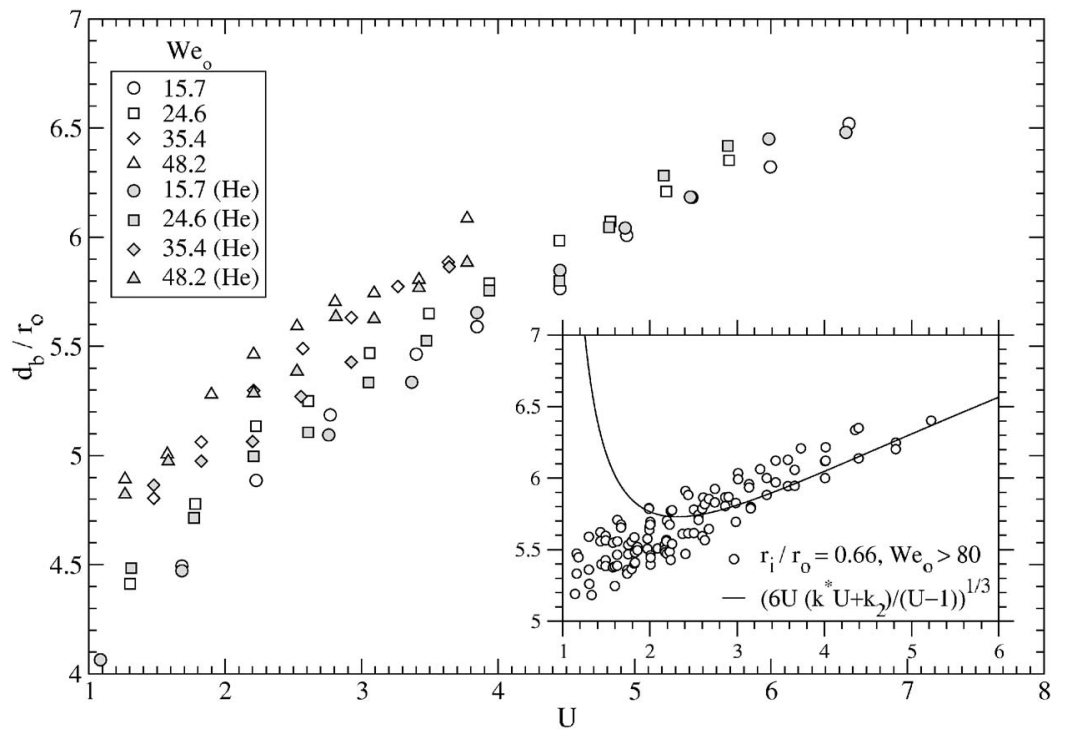

FIG. 13. Bubble equivalent diameters at constant flowrate conditions. Hollow symbols correspond to experiments performed with air and solid symbols indicate experiments performed with helium. The inset shows the experimental results obtained at $\mathrm{We}_{o} \gg 1$, together with the fit provided by Eq. (24), where $k^{*}=5.84$ and $k_{2}=4.29$.

quency, $6 / \tau_{b}$, with $U$ for different values of $\mathrm{We}_{o}$ at constant flow-rate conditions. Notice that the bubbling frequency increases as $U$ increases and the Weber number decreases. Moreover, the inset in Fig. 12 indicates that Eq. (24) predicts well the measured bubbling frequencies obtained experimentally for large values of $\mathrm{We}_{o}$ and $U>2$. However, Eq. (24) does not capture the behavior of the measured frequencies at low values of $U$. This is a consequence of the fact that, in this limit, the collapse stage is no longer clearly dominated by the negative pressure at the needle exit. Indeed, at low values of $U$ the capillary pressure becomes of the order of $\Pi_{e}$ and contribute equally to the inward acceleration of the neck. Figure 12 also displays that the bubble formation frequency does not significantly depend on the gas density for large values of $U$ as can be observed by comparing the results for air and helium at the same value of $\mathrm{We}_{o}$.

The equivalent bubble diameter, calculated as $d_{b}$ $=\left[6 Q_{i} /\left(\pi f_{b}\right)\right]^{1 / 3}$, with $f_{b}=1 / t_{b}$ being the measured bubbling frequency, has been represented in Fig. 13 as a function of the gas-to-liquid mean velocity ratio for different Weber numbers. Note that, consistently with Eq. (24), the equivalent bubble diameter is $d_{b} / r_{o} \simeq\left[6 U\left(k^{*} U+k_{2}\right) /(U-1)\right]^{1 / 3}$ for large values of $U$ and $\mathrm{We}_{o}$, with $d_{b} / r_{o} \sim 3.3 U^{1 / 3}$ for $U \gg 1$. At this point, it is important to emphasize that, under constant flow rate conditions, the bubbling frequency and, therefore, the bubble size neither depend on the gas properties nor on the gas feeding system. Therefore, the only parameters controlling the dimensionless bubble size are $\mathrm{We}_{o}, U$ and the geometrical ratio $r_{i} / r_{o}$. The influence of the gas density on the bubble size has been proven to be small and can be neglected. Indeed, gas density is only relevant to describe the final instants of pinch-off, which takes place in a time scale much smaller than the bubbling period. These conclusions differ from the results presented in the following section, where it will be shown that the type of gas used and the (a)

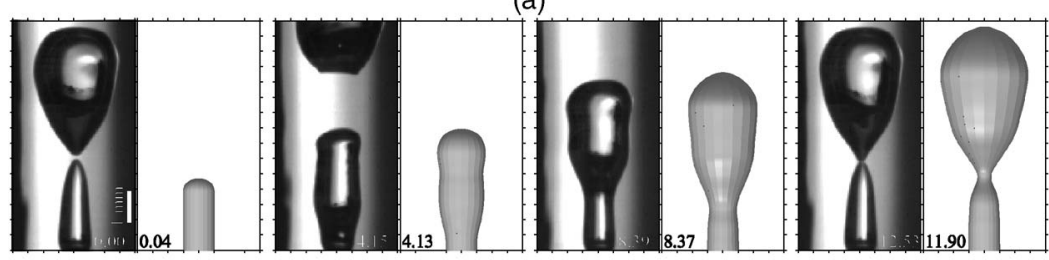

(b)

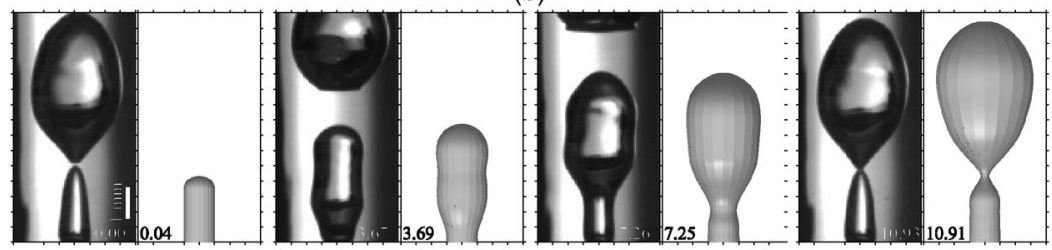

(c)

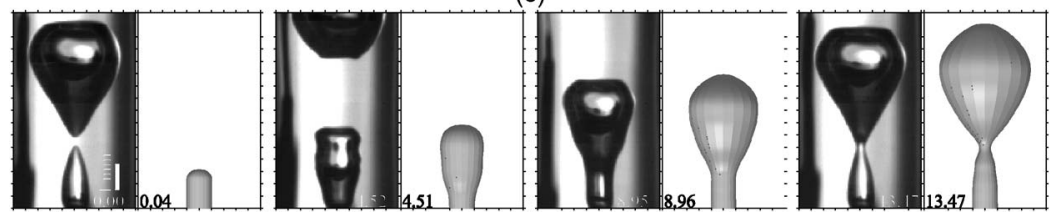

FIG. 14. Comparison of the experimental visualizations with the numerical simulations performed at constant pressure supply conditions for $\mathrm{We}_{o}=15.75$. (a) $U_{c}$ $=2.76, \quad\left(p_{c}-p_{a}\right) /\left(\rho_{o} u_{o}^{2}\right)=0.25, \quad(\mathrm{~b}) \quad U_{c}=3.87, \quad\left(p_{c}\right.$ $\left.-p_{a}\right) /\left(\rho_{o} u_{o}^{2}\right)=0.37$, and (c) $U_{c}=3.85,\left(p_{c}-p_{a}\right) /\left(\rho_{o} u_{o}^{2}\right)$ $=0.25$. The working gas is air in cases (a) and (b) and helium in case (c). The visualizations correspond to experimental set $\mathrm{X}$ in Table I. 

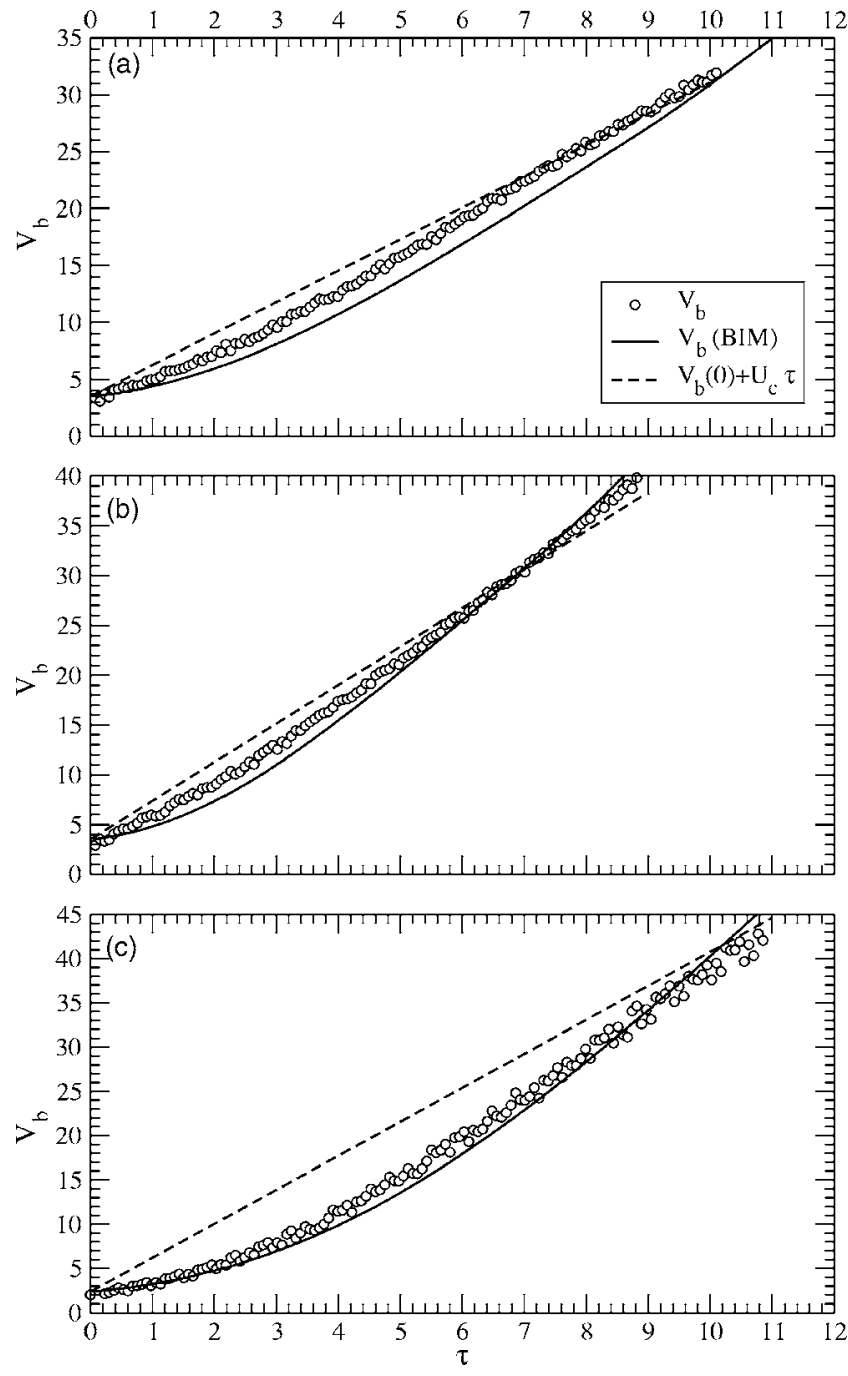

FIG. 15. Time evolution of the bubble volume at constant pressure supply conditions for $\mathrm{We}_{o}=15.75$. (a) $U_{c}=2.76,\left(p_{c}-p_{a}\right) /\left(\rho_{o} u_{o}^{2}\right)=0.25$, (b) $U_{c}$ $=3.87,\left(p_{c}-p_{a}\right) /\left(\rho_{o} u_{o}^{2}\right)=0.37$, and (c) $U_{c}=3.85,\left(p_{c}-p_{a}\right) /\left(\rho_{o} u_{o}^{2}\right)=0.25$. Symbols are the experimental measurements, solid lines indicate the numerical results, and dashed lines represent the time evolution of the volume that would take place at constant flow-rate conditions. The working gas is air in cases (a) and (b) and helium in case (c). The data correspond to experimental set $\mathrm{X}$ in Table I.

injection system may affect the bubble formation process under constant pressure supply injection conditions.

\section{B. Injection conditions at constant pressure supply}

This subsection provides experiments and numerical simulations where constant flow rate conditions are no longer ensured. The interest of this study relies on the fact that the cost of producing bubbles needs to be minimized in practical engineering applications, such as chemical reactors or fluidized beds. Indeed, injection at nonconstant flow rate conditions is given whenever the pressure drop from the gas reservoir to the needle exit is of the order of (or lower than) the pressure fluctuations at the needle exit, $\Delta p$. This contrasts with the much larger pressure drop along the feeding line which is usually necessary to ensure constant flow-rate conditions. In our case, the nonconstant flow rate experiments were performed using setup 2, with needles shorter than those used in the constant flow rate experiments (see Table I). As will be shown below, under the same operating conditions, i.e., $u_{o}$, the gas flow rate injected into the feeding chamber, $Q_{c}$, and $r_{o}$, the bubble formation frequencies obtained under constant pressure supply conditions are lower than their constant flow rate counterparts and, therefore, the equivalent diameter of the resulting bubbles is larger. Moreover, in the present case, the bubble formation frequency strongly depends on the pressure drop along the feeding line (the "flow resistance" in Oguz and Prosperetti ${ }^{19}$ ), indicating that the bubbling frequency and bubble size vary with the gas density, ${ }^{18}$ gas viscosity, and the geometry of the injection system. Thus, both the fact that the resulting bubbles are bigger than in the case of constant flow rate, and that the process depends on a large number of control parameters, constitute a drawback of these types of injection systems.

Figure 14 displays the experimental visualizations at constant pressure supply recorded for different values of the operating conditions and performed at $\mathrm{We}_{o}=15.75$ with the same injection needle (experimental set $\mathrm{X}$ in Table I). Figures 14(a) and 14(b) show the time evolution of an air bubble while Fig. 14(c) displays the evolution of a helium bubble at the same conditions given in Fig. 14(b). Since $Q_{i}=Q_{i}(t)$ $\neq Q_{c}$, the gas-to-liquid mean velocity ratio will be defined in this case as $U_{c}=Q_{c} /\left(\pi r_{o}^{2} u_{o}\right)$ rather than $U=Q_{i} /\left(\pi r_{o}^{2} u_{o}\right)$ used in the cases of constant flow-rate conditions. The fact that, although $Q_{c}$ remains constant during the bubbling process, the instantaneous velocity ratio, $Q_{i} /\left(\pi r_{o}^{2} u_{o}\right)$, varies with time is clearly observed in Fig. 15. Indeed, this figure shows that, at the initial stages of the bubble growth, $Q_{i}<Q_{c}$, while $Q_{i}$ $>Q_{c}$ at the final instants of the bubbling period. Note that the bubble volume evolutions of Fig. 15 are quite different from those shown in Fig. 7, where it can be observed that the experimental slopes of $V_{b}(\tau)$ remain constant.

Moreover, Fig. 16 shows that, under flow rate conditions that are not constant, the bubbling frequency decreases as the gas density decreases (helium bubbles). Also notice that, for a given gas, the bubbling frequency obtained at constant flow-rate conditions is considerably larger than that obtained at constant pressure supply conditions. The periodicity of bubble production implies that the averaged flow rate during a bubbling period is equal to the gas flow-rate injected into the chamber, $Q_{c}=V\left(t_{b}\right) / t_{b}$, where $t_{b}$ indicates the break-up time. Therefore, under the same operating conditions, $\left(\mathrm{We}_{o}\right.$, $U_{c}$ ) and needle radius, $r_{o}$, the size of the bubbles is smaller under constant flow-rate conditions than under constant pressure supply conditions. Moreover, under constant pressure supply, the air bubbles are consistently smaller than the helium bubbles. These conclusions are general and valid in the whole range of parameters explored, as will be discussed below.

Figure 14 also shows the comparison of the BIM numerical simulations with the experimental visualizations. The numerical simulations capture all the essential features of the bubble formation process (see Fig. 14) at the same time that reproduce the experimental time evolution of the bubble volume (see Fig. 15). In this case, the entries for the numerical simulation are just the Weber number and the dimensionless pressure at the needle exit in Eq. (A9) of the 

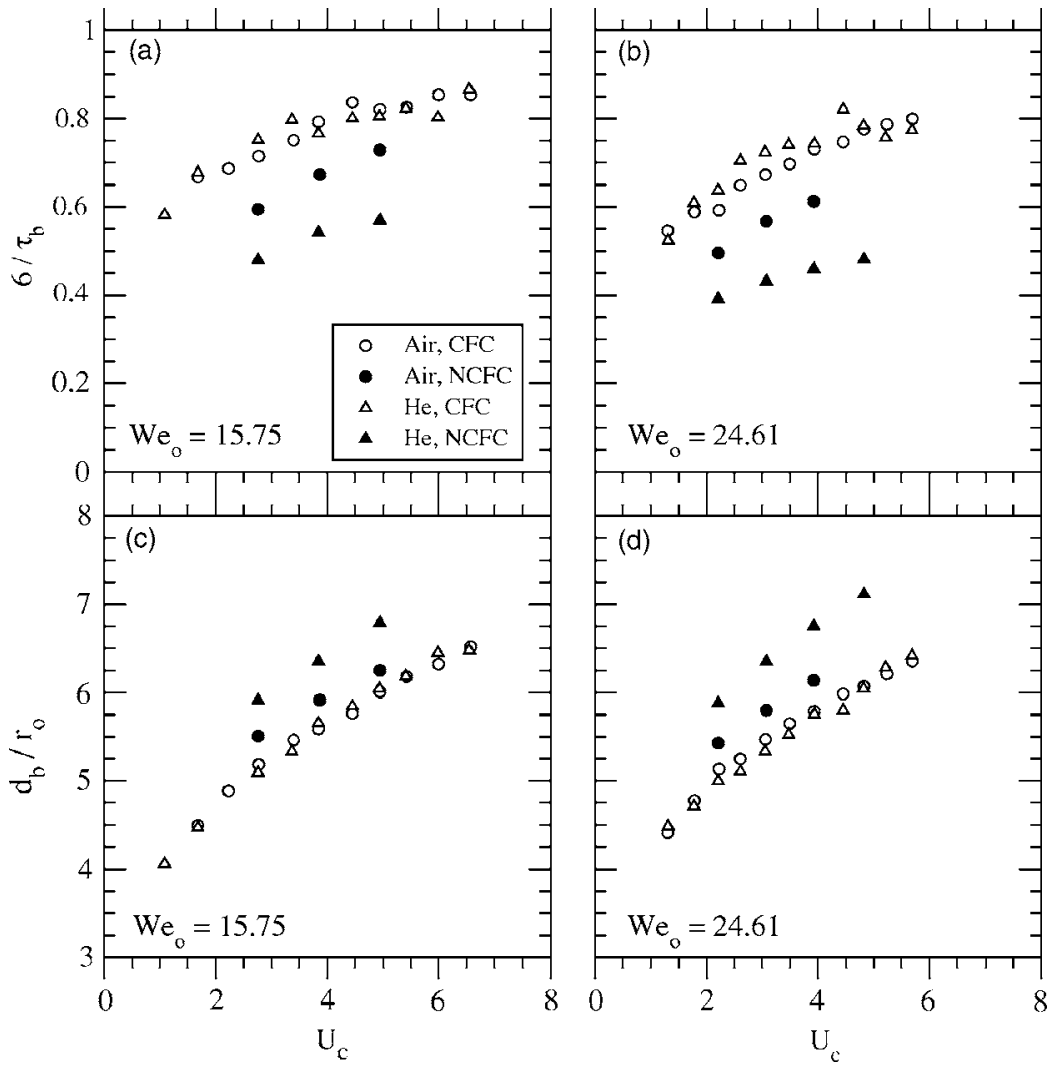

FIG. 16. Dependence of the bubbling frequency with $U_{c}$ for air (circles) and helium (triangles) at two different values of the Weber number, (a) $\mathrm{We}_{o}=15.75$ and (b) $\mathrm{We}_{o}=24.61$. The corresponding bubble diameter is shown in (c) and (d), respectively. Here CFC indicates constant flow-rate conditions and $\mathrm{NCFC}$ indicates nonconstant flow-rate conditions.

Appendix, $\Pi=\left(p_{c}-p_{a}\right) /\left(\rho_{o} u_{o}^{2}\right)-\Pi_{\text {line. }}$ The chamber pressure, $p_{c}$, was measured experimentally for each value of $Q_{c}$ and $u_{o}$. Similarly, $\Pi_{\text {line }}=p_{\text {line }} /\left(\rho_{o} u_{o}^{2}\right)$, with $p_{\text {line }}$ the pressure drop along the feeding line was also experimentally characterized and, for our particular experimental setup, can be given, in dimensionless form, as

$$
\begin{aligned}
\Pi_{\text {line }} & =\frac{1}{2} \frac{\rho_{i}}{\rho_{o}}\left(\frac{r_{o}}{r_{i}}\right)^{4} U_{c}^{2}(\tau)\left(3.9+\frac{16 l}{\operatorname{Re}_{i} r_{i}}\right) \\
& \sim \frac{1}{2} \frac{\rho_{i}}{\rho_{o}}\left(\frac{r_{o}}{r_{i}}\right)^{4} U_{c}^{2}(\tau) K\left(\operatorname{Re}_{i} r_{i} / l\right)
\end{aligned}
$$

with $\operatorname{Re}_{i}=Q_{i} /\left(\pi \nu_{i} r_{i}\right)$ the inner Reynolds number. During the development of this work, we verified that bubble formation strongly depended on $\Pi$. In fact, if $\Pi$ was not adequately determined for each particular experimental setup and gas properties, the agreement between experiments and numerics displayed in Fig. 14 would be lost. Consequently, in contrast to the case of injection at constant flow conditions, the bubble growth and detachment from a needle at constant pressure supply conditions depend on the injection system, gas density and gas viscosity. We have also checked that, consistently with Oguz and Prosperetti, ${ }^{19}$ as the pressure drop along the feeding line increases the volume of the bubbles obtained decreases. Moreover the results corresponding to constant flow rate conditions are recovered in the limit of $K\left(\operatorname{Re}_{i} r_{i} / l\right) \gg 1$.

Although the influence of the flow resistance on the size of the resulting bubbles was clearly shown by Oguz and Prosperetti, ${ }^{19}$ here we intend to explain, in very simple terms, the influence of the feeding line on the bubble expansion time. Note first that, assuming that the bubble is spherical, the bubble growth after the detachment of the previous one begins because $p_{c}-p_{a}-2 \sigma / r_{b}>0$, with $r_{b}$ the bubble radius. Consequently, the gas initially accelerates the liquid outwards, provoking the bubble surface to grow. Therefore, the pressure at the needle exit, $p_{\text {exit }}$, begins to decrease with time until it eventually drops below the pressure of the feeding chamber, $p_{c}$. This is caused by the fact that $p_{\text {exit }}=p_{c}$ $-1 / 2 \rho_{i} u_{i}^{2} \Pi_{\text {line }}$, where the gas velocity, $u_{i}$, is obtained using the continuity equation $\pi r_{o}^{2} u_{i}=4 \pi r_{b}^{2} d r_{b} / d t$. To conclude with this qualitative description, note that the expansion stage ends at the instant $t_{\exp }$ when the pressure at a point of the liquid stream on the bubble surface, $p_{\text {exit }}-2 \sigma / r_{b}$, drops below the pressure of the outer, surrounding liquid, $p_{a}$. Therefore, the liquid begins to accelerate inwards at the instant $t_{\exp }$ where $p_{\text {exit }}\left(t_{\text {exp }}\right)-2 \sigma / r_{b}\left(t_{\text {exp }}\right)=p_{a}$. With the aim at calculating the expansion time in a simplified manner, the time evolution of the bubble interface can be approached by means of the dimensionless Rayleigh-Plesset equation,

$$
\begin{aligned}
R \ddot{R}+\frac{3}{2} \dot{R}^{2}= & \frac{p_{c}-p_{a}}{\rho_{o} u_{o}^{2}}-\frac{2}{\mathrm{We}_{o} R} \\
& -\frac{1}{2} \frac{\rho_{i}}{\rho_{o}}\left(\frac{r_{o}}{r_{i}}\right)^{4}\left[4 R^{2} \dot{R}\right]^{2} K\left(\mathrm{Re}_{i} r_{i} / l\right),
\end{aligned}
$$

where $R=r_{b} / r_{o}$, and $K$ represents the pressure loss coefficient obtained from the experiments. In view of the above discussion, the condition which provides us with $\tau_{\exp }$ can be stated as 

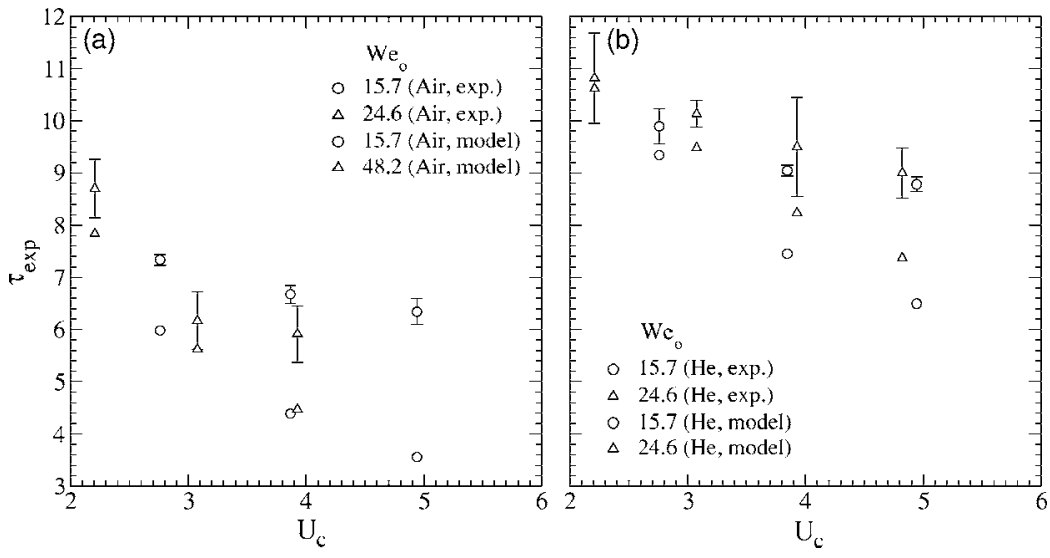

FIG. 17. Comparison of the dependence of the expansion time, $\tau_{\text {exp }}$, on the gas-to-liquid velocity ratio, $U_{c}$, given by the numerical simulations (filled symbols) and the experimental visualizations (hollow symbols) for several values of the Weber number, $\mathrm{We}_{o}$, for air (a) and helium (b). The error bars indicate the standard deviation of the measurements.

$$
\begin{aligned}
& \frac{p_{c}-p_{a}}{\rho_{o} u_{o}^{2}}-\frac{2}{\mathrm{We}_{o} R\left(\tau_{\exp }\right)} \\
& \quad-\frac{1}{2} \frac{\rho_{i}}{\rho_{o}}\left(\frac{r_{o}}{r_{i}}\right)^{4}\left[4 R^{2}\left(\tau_{\exp }\right) \dot{R}\left(\tau_{\exp }\right)\right]^{2} K\left(\mathrm{Re}_{i} r_{i} / l\right)=0,
\end{aligned}
$$

which can be calculated by solving Eq. (26) with the initial conditions $R(0)=1, \dot{R}(0)=0$.

Figure 17 shows the comparison between the expansion times calculated through Eqs. (26) and (27) and those determined experimentally. The results of the simple model described above quantitatively agree with the experimental measurements, with the differences being just a consequence of the distinct criterion used to determine the expansion time, $\tau_{\text {exp. }}$. In fact, while Eq. (27) indicates that the expansion stage finishes when the exit pressure is $p_{\text {exit }}=p_{a}+2 \sigma / r_{b}$, the experimental values were determined when the radius of the neck was $r_{n}=0.9 r_{g}$. Notice that when $p_{\text {exit }}=p_{a}+2 \sigma / r_{b}$ the outer liquid, which was moving radially outward, begins to slow down until eventually stops and starts to move toward the axis forming the neck in the gas ligament. This process takes a certain time that depends on the liquid inertia, justifying therefore the smaller values of $\tau_{\text {exp }}$ given by the model. Moreover, since the liquid inertia increases with $U_{c}$, the differences between the experimental values and those provided by the model also increase with $U_{c}$.

\section{CONCLUSIONS}

We have studied the mechanisms underlying the periodic formation of gas bubbles within a laminar, high Reynolds number liquid jet both experimentally and with the aid of boundary-integral numerical simulations. Two different experimental setups were considered in order to elucidate the role of the gas injection system. In addition, a detailed discussion of the differences between constant and nonconstant flow rate injection conditions is provided, including the requirements that a certain gas injection system needs to satisfy to guarantee a constant bubble injection flow-rate.

From a morphological point of view, the bubbles formed have different shapes depending on the Weber number; the bubbles display a shape similar to an almond at low Weber numbers while they look like a peanut for larger values of $\mathrm{We}_{o}$. Guided by the analysis of the numerical results the size of the resulting bubbles has been properly scaled for each of the two experimental setups analyzed as a function of the gas-to-liquid mean velocity ratio, and the gas-to-liquid density ratio $\rho_{i} / \rho_{o}$. More precisely, in the case of constant flowrate injection conditions, the bubbling frequency has been shown to hardly depend on the gas density, with a bubble size that, for sufficiently large values of $U$ and $\mathrm{We}_{o}$, scales as $d_{b} / r_{o} \sim U^{1 / 3}$.

In the case of constant pressure supply conditions, the bubble size strongly depends on the pressure drop along the feeding line and, consequently, on the density of the gas injected. This dependence makes the control of the bubble formation process considerably more difficult than in the constant flow-rate case. Furthermore, under the operating conditions reported here, the equivalent diameter of air and helium bubbles is, respectively, about $10 \%$ and $20 \%$ larger than the constant flow-rate counterpart. In addition, the experiments show that, under constant pressure supply, helium bubbles are approximately $10 \%$ larger than air bubbles due to the gas density effect on the bubbling process.

Another conclusion which can be extracted from the present work concerns the essential role of gas inertia in the description of the latest stages previous to pinch-off. Indeed, experiments and numerical simulations retaining gas inertia agree both in the geometry of the bubbles and in the time scale throughout the whole bubble formation process. However, simulations neglecting gas inertia do not reproduce the experimentally observed behavior at times close to pinch-off. These simulations show that the neck is still collapsing while in the experimental visualizations the bubble has already pinched-off. The experimental results indicate that, once the neck is formed, the velocity of the gas through it increases, the pressure decreases and, consequently, the neck collapses more rapidly due to a Venturi effect; a phenomenon not taken into account in this type of simulations. In addition, the evolution of the neck in the numerical computations carried out neglecting gas inertia is symmetric, in the sense that the shapes of the interface upstream and downstream of the neck are identical, while the experimental collapse takes place asymmetrically. Moreover, a final evidence of the effect of gas inertia on the final instants of the bubbling process has been reported analyzing the velocity of the liquid jets penetrating the forming bubble at the break-up moment. The experiments performed under the same operating conditions with air and helium indicated that the velocity of the pen- 


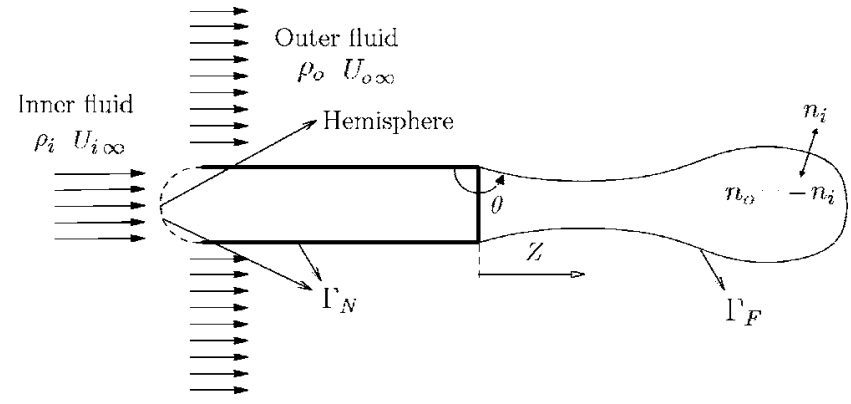

FIG. 18. Sketch of the flow configuration considered in the numerical simulations.

etrating jets were considerably smaller in the cases of helium bubbles than in the cases of air bubbles, which demonstrate the key role of gas inertia in jet formation.

\section{ACKNOWLEDGMENTS}

This work was supported by Spanish Ministry of Education under Projects Nos. DPI2005-08654-C04-01 and DPI2005-08654-C04-02. J.M.G. is grateful to H. A. Stone for his help in partially writing the Appendix and for his hospitality during his stay at Harvard University.

\section{APPENDIX: NUMERICAL PROCEDURE}

Consider the situation displayed in Fig. 18, of a gas flow at a constant velocity $u_{i}$ injected through a needle (inner fluid in Fig. 18) and discharging into another fluid (outer fluid in Fig. 18) whose velocity at infinity, $u_{o}$, is parallel to the injection needle and can be different from zero. The main difference between the present situation and that considered in Oguz and Prosperetti ${ }^{19}$ is that the procedure presented here permits us to take into account the inertia of both the inner and outer fluids. Here we provide an axisymmetric boundary integral method (BIM), based on that described in Rodríguez-Rodríguez et al., ${ }^{42}$ which is especially useful when both, the solid boundaries, and the free surface separating two fluids with different densities are present. For the problem at hand, the next set of parameters and dimensionless variables will be considered:

$$
\Phi_{(i, o)}=\frac{\phi_{(i, o)}}{u_{o} r_{o}}, \quad \tau=\frac{t u_{o}}{r_{o}}, \quad \mathrm{We}_{o}=\frac{\rho_{o} u_{o}^{2} r_{o}}{\sigma}, \quad U=\frac{u_{i}}{u_{o}},
$$

where $\Phi_{(i, o)}$ is the dimensionless potential, $\tau$ is the dimensionless time, $\mathrm{We}_{o}$ is the Weber number based on the outer stream and, since the needle is assumed to have zero thickness, $r_{i}=r_{o}, U$ is the inner-to-outer fluid velocity ratio. For numerical purposes, it proves convenient to divide the boundary separating the inner and outer fluids into $\Gamma_{N}$ and $\Gamma_{F}$, which are simply the needle surface and the free surface, respectively, with, $\Gamma=\Gamma_{N} \cup \Gamma_{F}$ (see Fig. 18). The following Green's formula, applied to each fluid, provides the velocity potentials $\Phi_{(i, o)}$ for the boundary positions $\mathbf{X}=(R, Z)$ :

$$
\begin{aligned}
\Phi_{(i, o)}(R, Z)= & S_{(i, o)}(R, Z)+\int_{\Gamma_{N} \cup \Gamma_{F}} g_{(i, o)} \frac{\partial \Phi_{(i, o)}}{\partial n_{(i, o)}} d s \\
& -\int_{\Gamma_{N} \cup \Gamma_{F}} h_{(i, o)} \Phi_{(i, o)} d s,
\end{aligned}
$$

where $g$ and $h$ are the kernel functions defined in Oguz and Prosperetti ${ }^{19}$ and Rodríguez-Rodríguez et al. ${ }^{42} \mathbf{n}_{(i, o)}$ is the normal vector pointing out the inner (outer) boundaries and $s$ is the arc length along the needle boundary and gas-liquid interface. Also notice that $S_{i}(R, Z)=0$ and $S_{o}=2 U Z$.

Since the boundary has been divided in two, the discretized versions of the kernel functions $g_{(i, o)}$ and $h_{(i, o)}$ in Eq. (A2), $\mathbf{g}_{(i, o)}$ and $\mathbf{h}_{(i, o)}$ (see Rodríguez-Rodríguez et al. ${ }^{42}$ ) can be written, in matrix form, as

$$
\mathbf{g}_{(i, o)}=\left[\frac{\mathbf{G}_{(i, o)}^{N N}}{\mathbf{G}_{(i, o)}^{F N}} \frac{\mathbf{G}_{(i, o)}^{N F}}{\mathbf{G}_{(i, o)}^{F F}}\right], \quad \mathbf{h}_{(i, o)}=\left[\frac{\mathbf{h}_{(i, o)}^{N N}}{\mathbf{h}_{(i, o)}^{F N}} \frac{\mathbf{h}_{(i, o)}^{N F}}{\mathbf{h}_{(i, o)}^{F F}}\right] .
$$

It needs to be pointed out that the needle surface is impermeable for the outer fluid, and $\partial \Phi_{o} / \partial n_{o}=0$ along $\Gamma_{N}$. Similarly, $\Gamma_{N}$ is also impermeable with respect to the inner fluid except at the hemisphere placed upstream of the needle where the inflow condition is imposed $\left(\partial \Phi_{i} / \partial n_{i} \neq 0\right)$ as shown in Fig. 18.

The unknowns of the integral equation (A2) are the potentials along the inner and outer sides of the needle $\left(\Phi_{i}^{N}\right.$ and $\Phi_{o}^{N}$ respectively), the potentials along the free surface $\left(\Phi_{i}^{F}\right.$ and $\Phi_{o}^{F}$ ), and the normal velocity at the free surface $\left(\partial \Phi_{i}^{F} / \partial n_{i}\right)$ Thus, the discretization of integral equation (A2) provides the following system of equations:

$$
\begin{aligned}
& \mathbf{G}_{i}^{N F} \frac{\partial \mathbf{\Phi}_{i}^{F}}{\partial n}-\mathbf{H}_{i}^{N F} \mathbf{\Phi}_{i}^{F}-\mathbf{H}_{i}^{N N} \mathbf{\Phi}_{i}^{N}=-\mathbf{G}_{i}^{N N} \frac{\partial \mathbf{\Phi}_{i}^{N}}{\partial n}, \\
& \mathbf{G}_{i}^{F F} \frac{\partial \mathbf{\Phi}_{i}^{F}}{\partial n}-\mathbf{H}_{i}^{F F} \boldsymbol{\Phi}_{i}^{F}-\mathbf{H}_{i}^{F N} \boldsymbol{\Phi}_{i}^{N}=-\mathbf{G}_{i}^{F N} \frac{\partial \boldsymbol{\Phi}_{i}^{N}}{\partial n}, \\
& -\mathbf{G}_{o}^{N F} \frac{\partial \mathbf{\Phi}_{i}^{F}}{\partial n}-\left(\rho_{i} / \rho_{o}\right) \mathbf{H}_{o}^{N F} \boldsymbol{\Phi}_{i}^{F}-\mathbf{H}_{o}^{N N} \mathbf{\Phi}_{o}^{N}=\mathbf{S}^{N}+\mathbf{H}_{o}^{N F} \mathbf{\Psi}, \\
& -\mathbf{G}_{o}^{F F} \frac{\partial \mathbf{\Phi}_{i}^{F}}{\partial n}-\left(\rho_{i} / \rho_{o}\right) \mathbf{H}_{o}^{F F} \boldsymbol{\Phi}_{i}^{F}-\mathbf{H}_{o}^{F N} \boldsymbol{\Phi}_{o}^{N}=\mathbf{S}^{F}+\mathbf{H}_{o}^{F F} \mathbf{\Psi},
\end{aligned}
$$

with $\mathbf{H}^{I J}=\mathbf{I}+\mathbf{h}^{I J}$ and $\mathbf{S}=-2 U \mathbf{Z}$. Note, that in Eqs. (A4) we have made use of the definition

$$
\boldsymbol{\Psi}=\boldsymbol{\Phi}_{o}^{F}-\rho_{i} / \rho_{o} \boldsymbol{\Phi}_{i}^{F},
$$

where the modified potential $\psi$ in Eq. (A5) is analogous to that defined in Rodríguez-Rodríguez et $a .^{42}$ Moreover, to deduce Eqs. (A4), we have taken into account the fact that $\mathbf{g}_{o}=\mathbf{g}_{i}$ and $\partial \Phi_{i} / \partial n_{i}=-\partial \Phi_{o} / \partial n_{o}$. The vector $\Phi_{o}^{F}$ is obtained from Eq. (A5) once $\Phi_{i}^{F}$ is determined from the solution of the system (A4). The only additional numerical detail that needs to be addressed with respect to the numerical procedure described in Rodríguez-Rodríguez et al. ${ }^{42}$ is that the node at the lip of the needle, which belongs simultaneously to both the needle and the free surface boundaries, is included in the needle matrices (the ones that contain the su- 
perscript $N$ ). Furthermore, for this special point, the left-hand side of Eq. (A2) is multiplied by $\theta / \pi$ in the case of the internal fluid and by $(2 \pi-\theta) / \pi$ in the case of the external fluid (see Fig. 18). In terms of the dimensionless variables and parameters defined in Eq. (A1), the equation for the time evolution of the modified potential $\psi=\Phi_{o}-\left(\rho_{i} / \rho_{o}\right) \Phi_{i}$ reads

$$
\begin{aligned}
\frac{D^{n} \psi}{D \tau}= & P_{0 o}-P_{0 i}+\mathrm{We}_{o}^{-1} \mathcal{C}+\frac{1}{2}\left(1-\frac{\rho_{i}}{\rho_{o}}\right)\left(\frac{\partial \Phi}{\partial n}\right)^{2} \\
& -\frac{1}{2}\left[\left(\frac{\partial \Phi_{o}}{\partial s}\right)^{2}-\frac{\rho_{i}}{\rho_{o}}\left(\frac{\partial \Phi_{i}}{\partial s}\right)^{2}\right],
\end{aligned}
$$

where $P_{0 o}$ and $P_{0 i}$ are the outer and inner stagnation pressures, respectively, and $\mathcal{C}=\nabla \cdot \mathbf{n}_{i}$ indicates the surface curvature. Note that in the Bernoulli equation (A6),

$$
\frac{\mathrm{D}^{n} \psi}{\mathrm{D} \tau}=\frac{\partial \psi}{\partial \tau}+\left(1-\frac{\rho_{i}}{\rho_{o}}\right)\left(\frac{\partial \Phi}{\partial n}\right)^{2}
$$

is the material derivative of $\psi$ along the normal to the interface and that $\left(\partial \phi_{i} / \partial n_{i}\right)^{2}=\left(\partial \phi_{o} / \partial n_{o}\right)^{2}=(\partial \phi / \partial n)^{2}$. In the following we will assume that $P_{0 o}-P_{0 i}=1 / 2\left(1-\rho_{i} / \rho_{o} U^{2}\right)$ $-\mathrm{We}_{o}^{-1}$, a function which is independent of time and realistic, since it corresponds to the difference of pressure corresponding to a cylindrical free surface at the exit of the injection needle. As already mentioned in the main body of the paper, simulations neglecting the gas convective terms in Eq. (A6) were also performed. In those cases, Eqs. (4) were solved together with

$$
\frac{D^{n} \psi}{D \tau}=\frac{1}{2}+\mathrm{We}_{o}^{-1}(\mathcal{C}-1)+\frac{1}{2}\left(\frac{\partial \Phi}{\partial n}\right)^{2}-\frac{1}{2}\left(\frac{\partial \Phi_{o}}{\partial s}\right)^{2} .
$$

In addition, we also performed some simulations where the gas was injected at a constant pressure supply conditions, rather than at a constant flow-rate conditions. In those cases, for simplicity, the gas inertia within the bubble was also neglected and, therefore, the corresponding Bernoulli equation for the outer flow potential should be given by

$$
\frac{D^{n} \Phi_{o}}{D \tau}=\frac{1}{2}-\Pi+\mathrm{We}_{o}^{-1} \mathcal{C}+\frac{1}{2}\left(\frac{\partial \Phi}{\partial n}\right)^{2}-\frac{1}{2}\left(\frac{\partial \Phi_{o}}{\partial s}\right)^{2},
$$

where $\Pi=\left(p_{c}-p_{a}\right) /\left(\rho_{o} u_{o}^{2}\right)-\Pi_{\text {line }}, p_{c}$ is the chamber pressure and $\Pi_{\text {line }}=\Pi_{\text {line }}\left(R e_{i} r_{i} / l\right)$ indicates the dimensionless pressure loss along the feeding line. Note that the gas velocity entering the bubble (at the needle exit) is calculated as

$$
u_{i}=\frac{d V_{b} / \pi}{d \tau},
$$

with $V_{b}$ being the bubble volume. Furthermore, the liquid normal velocity at the bubble free surface together with the liquid potential along the needle surface are obtained by solving the system

$$
\begin{aligned}
& -\mathbf{G}_{o}^{N F} \frac{\partial \Phi_{i}^{F}}{\partial n}-\mathbf{H}_{o}^{N N} \Phi_{o}^{N}=\mathbf{S}^{N}+\mathbf{H}_{o}^{N F} \Phi_{o}^{F}, \\
& -\mathbf{G}_{o}^{F F} \frac{\partial \Phi_{i}^{F}}{\partial n}-\mathbf{H}_{o}^{F N} \Phi_{o}^{N}=\mathbf{S}^{F}+\mathbf{H}_{o}^{F F} \Phi_{o}^{F}
\end{aligned}
$$

In the numerical implementation reported here, we considered an injection needle of length 15 , an initial bubble shape given by a cylinder ended by a hemisphere, and the numerical intact length, $l_{i}$, taken from our experimental measurements. Note that the nodes used to discretize the needle surface were not equally distributed along its length, but they were clustered near the exit. ${ }^{19}$ Moreover, the arc length of the elements used to discretize the free surface was kept constant and equal to $5 \times 10^{-2}$ by adding more nodes as the length of $\Gamma_{N}$ increased with time. For the cases of constant flow-rate, the inner axial velocity along the permeable hemisphere of $\Gamma_{N}$ (see Fig. 18), was varied with time as $1-(1-U)$ $\times \exp (-5 \tau)$. Consequently, except for a very short initial transient, the injected flow rate remained constant during the numerical simulations.

${ }^{1}$ W. S. Rayleigh, "On the instability of jets," Proc. London Math. Soc. 10, 4 (1878)

${ }^{2}$ F. Savart, "Mémoire sur la constitution des veines liquides lancées par des orifices circulaires en mince paroi," Ann. Chim. Phys. 53, 337 (1833).

${ }^{3}$ J. Plateau, Statique Expérimentale et Théorique des Liquides (GauthierVillars et $\mathrm{C}^{i e}$, Paris, 1873).

${ }^{4}$ J. Eggers, "Nonlinear dynamics and breakup of free surface flows," Rev. Mod. Phys. 69, 865 (1997).

${ }^{5}$ C. Clanet and J. C. Lasheras, "Transition from dripping to jetting," J. Fluid Mech. 383, 307 (1999).

${ }^{6}$ B. Ambravaneswaran, H. J. Subramani, S. D. Phillips, and O. A. Basaran, "Dripping-jetting transitions in a dripping faucet," Phys. Rev. Lett. 93, 034501 (2004)

${ }^{7}$ P. Doshi, I. Cohen, W. W. Zhang, M. Siegel, P. Howell, O. A. Basaran, and S. R. Nagel, "Persistence of memory in drop breakup: The breakdown of universality," Science 302, 1185 (2003).

${ }^{8}$ R. Suryo, P. Doshi, and O. A. Basaran, "Non-self-similar, linear dynamics during pinch-off of a hollow annular jet," Phys. Fluids 16, 4177 (2004).

${ }^{9}$ J. Burton, R. Waldrep, and P. Taborek, "Scaling instabilities in bubble pinch-off," Phys. Rev. Lett. 94, 184502 (2005).

${ }^{10}$ D. Leppinen and J. R. Lister, "Capillary pinch-off in inviscid fluids," Phys. Fluids 15, 568 (2003).

${ }^{11}$ J. M. Gordillo, A. Sevilla, J. Rodríguez-Rodríguez, and C. MartínezBazán, "Axisymmetric bubble pinch-off at hig Reynolds numbers," Phys. Rev. Lett. 95, 194501 (2005).

${ }^{12}$ R. Bergmann, D. Meer, M. Stijnman, M. Sandtke, A. Prosperetti, and D. Lohse, "Giant bubble pinch-off," Phys. Rev. Lett. 96, 154505 (2006).

${ }^{13}$ N. C. Keim, P. Moller, W. W. Zhang, and S. R. Nagel, "Breakup of air bubbles in water: Memory and breakdown of cylindrical symmetry," Phys. Rev. Lett. 97, 144503 (2006).

${ }^{14}$ J. F. Davidson and B. O. G. Schuler, "Bubble formation at an orifice in an inviscid liquid," Trans. Inst. Chem. Eng. 38, 335 (1960).

${ }^{15}$ R. Kumar and N. R. Kuloor, "The formation of bubbles and drops," Adv. Chem. Eng. 8, 256 (1970).

${ }^{16}$ A. Marmur and E. Rubin, "A theoretical model for bubble formation at an orifice submerged in an inviscid liquid," Chem. Eng. Sci. 31, 453 (1976).

${ }^{17}$ R. B. H. Tan and I. J. Harris, "A model for non-spherical bubble growth at a single orifice," Chem. Eng. Sci. 41, 3175 (1986).

${ }^{18}$ P. M. Wilkinson and L. L. van Dierendonck, "A theoretical model for the influence of gas properties and pressure on single-bubble formation at an orifice," Chem. Eng. Sci. 49, 1429 (1994).

${ }^{19}$ H. N. Oguz and A. Prosperetti, "Dynamics of bubble growth and detachment from a needle," J. Fluid Mech. 257, 111 (1993).

${ }^{20} \mathrm{~K}$. Terasaka and H. Tsuge, "Bubble formation under constant flow conditions," Chem. Eng. Sci. 48, 3417 (1993).

${ }^{21}$ F. J. Higuera and A. Medina, "Injection and coalescence of bubbles in a 
quiescent inviscid liquid,” Eur. J. Mech. B/Fluids 25, 164 (2006).

${ }^{22}$ J. M. Gordillo, A. M. Gañán-Calvo, and M. Pérez-Saborid, "Monodisperse microbubbling: Absolute instabilities in coflowing gas-liquid jets," Phys. Fluids 13, 3839 (2001).

${ }^{23}$ A. Sevilla, J. M. Gordillo, and C. Martínez-Bazán, "Transition from bubbling to jetting in a coaxial air-water jet," Phys. Fluids 17, 018105 (2005).

${ }^{24} \mathrm{H}$. Tsuge, Encyclopedia of Fluid Mechanics (Gulf, Houston, Texas, 1986).

${ }^{25}$ F. Johnsson, G. Larsson, and B. Leckner, "Pressure and flow fluctuations in a fluidized bed-interaction with the air-feed system," Chem. Eng. Sci. 57, 1379 (2002)

${ }^{26}$ M. Kukizaki and M. Goto, "Size control of nanobubbles generated from shirasu-porous-glass (spg) membranes," J. Membr. Sci. 281, 386 (2006)

${ }^{27}$ S. C. Chuang and V. W. Goldschmidt, "Bubble formation due to a submerged capillary tube in quiescent and coflowing streams," ASME J. Basic Eng. 92, 705 (1970).

${ }^{28}$ H. A. Stone, A. D. Stroock, and A. Adjari, "Engineering flows in small devices: Microfluidics toward a lab-on-a-chip," Annu. Rev. Fluid Mech. 36, 381 (2004).

${ }^{29}$ A. M. Gañán-Calvo and J. M. Gordillo, "Perfectly monodisperse microbubbling by capillary flow focusing," Phys. Rev. Lett. 87, 274501 (2001).

${ }^{30}$ J. M. Gordillo, Z. Cheng, M. Márquez, A. M. Gañán-Calvo, and D. A. Weitz, "A new device for the generation of microbubbles," Phys. Fluids 16, 2828 (2004)

${ }^{31}$ T. Cubaud and C. M. Ho, "Transport of bubbles in square microchannels," Phys. Fluids 16, 4575 (2004).

${ }^{32}$ P. Garstecki, I. Gitlin, W. DiLuzio, G. M. Whitesides, E. Kumacheva, and H. A. Stone, "Formation of monodisperse bubbles in a microfluidic flow- focusing device," Appl. Phys. Lett. 85, 2649 (2004).

${ }^{33}$ P. Garstecki, H. A. Stone, and G. M. Whitesides, "Mechanism for flowrate controlled breakup in confined geometries: A route to monodisperse emulsions," Phys. Rev. Lett. 94, 164501 (2005).

${ }^{34}$ E. Lorenceau, Y. Y. C. Sang, R. Hohler, and S. Cohen-Addad, "A high rate flow-focusing foam generator," Phys. Fluids 18, 097103 (2006).

${ }^{35}$ A. M. Gañán-Calvo, "Perfectly monodisperse microbubbling by capillary flow focusing: An alternate physical description and universal scaling," Phys. Rev. E 69, 027301 (2004).

${ }^{36}$ M. Cubaud, T. Tatieni, X. Zhong, and C. M. Ho, "Bubble dispenser in microfluidic devices," Phys. Rev. E 72, 037302 (2005).

${ }^{37}$ M. J. Jensen, H. A. Stone, and H. Bruus, "A numerical study of two-phase Stokes flow in an axisymmetric flow-focusing device," Phys. Fluids 18, 077103 (2006).

${ }^{38}$ A. Gañán-Calvo, M. Herrada, and P. Garstecki, "Bubbling in unbounded coflowing liquids," Phys. Rev. Lett. 96, 124504 (2006).

${ }^{39}$ A. Sevilla, J. M. Gordillo, and C. Martínez-Bazán, "Bubble formation in a coflowing air-water stream," J. Fluid Mech. 530, 181 (2005).

${ }^{40}$ D. B. Mcleod, "Relation between surface tension and density," Trans. Faraday Soc. 19, 38 (1923).

${ }^{41}$ A. M. Worthington, A Study of Splashes (Longman and Green, London, 1908).

${ }^{42}$ J. Rodríguez-Rodríguez, J. M. Gordillo, and C. Martínez-Bazán, "Breakup time and morphology of drops and bubbles in a high Reynolds number flow," J. Fluid Mech. 548, 69 (2006).

${ }^{43}$ J. M. Gordillo and M. A. Fontelos, "Satellites in the inviscid breakup of bubbles," Phys. Rev. Lett. 98, 144503 (2007). 